\title{
BMJ Open Targeting intensive versus conventional glycaemic control for type 1 diabetes mellitus: a systematic review with meta-analyses and trial sequential analyses of randomised clinical trials
}

\author{
Pernille Kähler, ${ }^{1}$ Berit Grevstad, ${ }^{1}$ Thomas Almdal, ${ }^{2}$ Christian Gluud, ${ }^{1,3}$ \\ Jørn Wetterslev, ${ }^{1}$ Allan Vaag, ${ }^{4}$ Bianca Hemmingsen ${ }^{1}$
}

To cite: Kähler P, Grevstad B, Almdal T, et al. Targeting intensive versus conventional glycaemic control for type 1 diabetes mellitus: a systematic review with meta-analyses and trial sequential analyses of randomised clinical trials. BMJ Open 2014;4:e004806. doi:10.1136/bmjopen-2014004806

- Prepublication history and additional material is available. To view please visit the journal (http://dx.doi.org/ 10.1136/bmjopen-2014004806).

Received 7 January 2014 Revised 29 June 2014 Accepted 1 July 2014

CrossMark

For numbered affiliations see end of article.

Correspondence to Pernille Kähler; pernille_kahler@yahoo.dk

\section{ABSTRACT}

Objective: To assess the benefits and harms of targeting intensive versus conventional glycaemic control in patients with type 1 diabetes mellitus.

Design: A systematic review with meta-analyses and trial sequential analyses of randomised clinical trials.

Data sources: The Cochrane Library, MEDLINE, EMBASE, Science Citation Index Expanded and LILACS to January 2013.

Study selection: Randomised clinical trials that prespecified different targets of glycaemic control in participants at any age with type 1 diabetes mellitus were included.

Data extraction: Two authors independently assessed studies for inclusion and extracted data.

Results: 18 randomised clinical trials included 2254 participants with type 1 diabetes mellitus. All trials had high risk of bias. There was no statistically significant effect of targeting intensive glycaemic control on all-cause mortality (risk ratio $1.16,95 \% \mathrm{Cl} 0.65$ to 2.08 ) or cardiovascular mortality $(0.49,0.19$ to 1.24$)$. Targeting intensive glycaemic control reduced the relative risks for the composite macrovascular outcome $(0.63,0.41$ to $0.96 ; p=0.03)$, and nephropathy $(0.37,0.27$ to 0.50 ; $p<0.00001$. The effect estimates of retinopathy, ketoacidosis and retinal photocoagulation were not consistently statistically significant between random and fixed effects models. The risk of severe hypoglycaemia was significantly increased with intensive glycaemic targets $(1.40,1.01$ to 1.94$)$. Trial sequential analyses showed that the amount of data needed to demonstrate a relative risk reduction of $10 \%$ were, in general, inadequate. Conclusions: There was no significant effect towards improved all-cause mortality when targeting intensive glycaemic control compared with conventional glycaemic control. However, there may be beneficial effects of targeting intensive glycaemic control on the composite macrovascular outcome and on nephropathy, and detrimental effects on severe hypoglycaemia. Notably, the data for retinopathy and ketoacidosis were inconsistent. There was a severe lack of reporting on patient relevant outcomes, and all trials had poor bias control.

\section{Strengths and limitations of this study}

- The systematic review is based on a published protocol.

- We included 18 randomised clinical trials from a comprehensive search with no language limitations or restrictions on outcomes reported in the trials.

- The available evidence was evaluated with trial sequential analysis and sensitivity analysis.

- All trials had a high risk of bias.

- The trials lacked reporting on patient relevant outcomes.

\section{INTRODUCTION}

Patients with type 1 diabetes mellitus are at increased risk of developing microvascular and macrovascular complications, as well as an increased risk of all-cause mortality compared with the background population. ${ }^{1}$ Observational studies suggest that reduction of blood glucose levels in patients with type 1 diabetes mellitus is associated with lower risk of vascular complications. ${ }^{2}$ A large randomised clinical trial, the Diabetes Control and Complications Trial (DCCT),${ }^{3-46}$ suggested a beneficial effect of strict glycaemic control on the risk of primarily microvascular complications in patients with type 1 diabetes mellitus. Since the completion of the DCCT, $^{3-46}$ the patients included have been followed in an observational study (Epidemiology of Diabetes Interventions and Complications (EDIC)). Based on this study it has been generally accepted that tight glycaemic control should be the preferred glycaemic approach for patients with type 1 diabetes mellitus, in order to reduce the risk of complications and death. ${ }^{47}{ }^{48}$ Since the 
publication of the results of the DCCT, ${ }^{3-46}$ no large scale trials have been conducted challenging this approach.

The treatment recommendations for patients with type 1 diabetes mellitus are to a large extent based on the DCCT. ${ }^{3-46}$ There is currently no up-to date comprehensive systematic review investigating the benefits and harms of targeting intensive glycaemic control compared with conventional glycaemic control in randomised clinical trials, regardless of the length of intervention and the age of participants. Intensive glycaemic control may cause increased risk of hypoglycaemia. In addition, achieving intensive glycaemic control in patients with type 1 diabetes mellitus typically requires markedly increased efforts of the individual patient as well as the use of increased resources from the healthcare system, due to additional doctor visits, glucose measurements and insulin injections. ${ }^{49}$

The definition of intensive glycaemic control varies among trials and guidelines. The DCCT ${ }^{3-46}$ applied an intensive intervention target of glycosylated haemoglobin Alc $\left(\mathrm{HbA}_{1 \mathrm{c}}\right)<6.05 \%,{ }^{3-46}$ whereas the intensive target was $\mathrm{HbA}_{1 \mathrm{c}}$ $<7.5 \%$ in the Microalbuminuria Trial. ${ }^{50} 51$ The guidelines also lack consistency. The American Diabetes Association recommends a $\mathrm{HbA}_{1 \mathrm{c}}$ level for patients with type 1 diabetes mellitus of less than $7 \%{ }^{47}$ whereas the International Diabetes Federation recommends less than $6.5 \%{ }^{48}$

This systematic review combines current evidence from randomised clinical trials on the effect of targeting intensive glycaemic control versus conventional glycaemic control on all-cause mortality, cardiovascular mortality, cardiovascular disease, microvascular disease, cancer, body mass index, weight, adverse events, mild and severe hypoglycaemia, costs of intervention, quality of life and ketoacidosis in patients with type 1 diabetes mellitus.

\section{METHODS}

This review follows the recommendations of The Cochrane Collaboration, ${ }^{52}$ and is based on a published protocol. ${ }^{53}$ We included all randomised clinical trials with prespecified different targets of glycaemic control in participants at any age with type 1 diabetes mellitus.

\section{Search strategy}

We searched in The Cochrane Library, Medline, EMBASE, Science Citation Index Expanded, and LILACS in January 2013 for randomised clinical trials of targeting intensive glycaemic control versus conventional glycaemic control in patients with type 1 diabetes mellitus. Web appendix 1 describes the search strategies for each database. We also searched abstracts present at the American Diabetes Association and the European Association for the Study of Diabetes congresses. We searched reference lists of the included trials in (systematic) reviews and meta-analyses and health technology assessment reports. Clinicaltrials.gov was searched for trial protocols, unpublished data and ongoing trials. We performed internet searches for all trials, as well as contacted authors for information about additional trials.

\section{Study selection}

Two authors (PK and $\mathrm{BH}$ or $\mathrm{BG}$ ) independently screened titles and abstracts according to the inclusion criteria. We included a trial if it was a randomised clinical trial; compared targeting intensive glycaemic control versus conventional glycaemic control; and undertaken in patients with type 1 diabetes mellitus. Trials only including pregnant patients were excluded. We included trials irrespective of duration, language, publication status and predefined outcomes.

In the published protocol, we predefined inclusion of all trials comparing patients treated to a specific target for intensive glycaemic control with patients treated to a conventional target. ${ }^{53}$ The intensive glycaemic targets varied among the trials, but all the included trials compared the results of aiming at a distinct lower target compared with the target of the control group. That is, trials investigated the effect of the use of more versus less intensive glucose targets in patients with type 1 diabetes mellitus, irrespective of differences among trials in predefined targets and achieved glycaemic control. Trials investigating the effect of different insulin regimens without a predefined difference in terms of glycaemic targets between groups were therefore excluded.

\section{Data extraction and risk of bias assessment}

Two authors (PK and BH or BG) independently extracted information from each included trial by using standard data extraction forms, and assessed the risk of bias as defined in The Cochrane Handbook of Systematic Reviews of Interventions. ${ }^{52}$ We assessed the following risk of bias domains: sequence generation, concealment of allocation, blinding, incomplete outcome data, selective outcome reporting, academic bias and sponsor bias. ${ }^{52}{ }^{53}$ For each domain, bias control was classified as adequate, unclear or inadequate. Owing to the nature of the design of comparing intensive glycaemic targets versus conventional glycaemic targets, it is not possible to blind the healthcare providers and the patients. Blinding was considered adequate if the outcome assessors were blinded. As most trials were with a high risk of bias, we divided the trials into those with a lower risk of bias, and those with a high risk of bias based on assessment of sequence generation, concealment of allocation, and blinding (table 4) ${ }^{52}{ }^{53}$ When we judged all three domains to be of low risk of bias, we designated the trial as having a lower risk of bias.

Discrepancies between the initial two authors' assessments were resolved by involvement of a third author (BH or BG). We extracted data at a trial level on several baseline characteristics of the participants (such as age, duration of disease and $\mathrm{HbA}_{1 \mathrm{c}}$ ), and outcomes. The predefined primary outcomes reported in this review were $^{53}$ all-cause mortality; cardiovascular mortality; and severe adverse events. Our secondary outcomes were ${ }^{53}$ : 
macrovascular complications (reported as a composite outcome); non-fatal myocardial infarction; non-fatal stroke, amputation of lower extremity; cardiac or peripheral revascularisation; mild and severe hypoglycaemia; microvascular complications (reported as a composite outcome); retinopathy; nephropathy; retinal photocoagulation; end-stage renal disease, cancer, congestive heart failure, ketoacidosis; weight/body mass index (BMI); health-related quality of life, and cost of interventions. We sought any relevant missing information from the original author(s) of the trial. When we identified more than one publication of an original trial, we assessed these together to maximise data collection. In case of substantial disagreement between older and newer publications, we contacted the authors. Data were extracted for both the end of the intervention period (the active treatment phase) and to the longest follow-up, if the trial had an observational follow-up period beginning after the active treatment phase.

\section{Statistical analyses}

We used Review Manager V.5.2 $2^{54}$ for statistical analyses. We summarised dichotomous data as relative risks with 95\% CIs. We used a random effects model and a fixed effect model. ${ }^{55}{ }^{56}$ In case of discrepancy between the two models, we reported both results; otherwise, we reported the random effects model. We examined heterogeneity with the $\mathrm{I}^{2}$ statistic. The data were analysed according to the intention-to-treat analysis.

We planned the following subgroup analyses for the primary outcomes: trials with a high risk of bias compared to trials with a lower risk of bias; published trials compared to unpublished trials; the use of human insulin compared to the use of insulin analogues; trials including participants at all ages compared to trials including participants older than 18 years.

Data were cross checked with previous meta-analyses ${ }^{57-61}$ and additional data could be included from three meta-analyses. ${ }^{57-59}$

We conducted trial sequential analyses. ${ }^{62} 63$ This is similar to interim analyses in a single trial, where monitoring boundaries are used to decide whether a trial could be terminated early when a $p$ value is sufficiently small to show the anticipated effect. Because there is no reason why the standards for a meta-analysis should be less rigorous than those for a single trial, analogous trial sequential monitoring boundaries can be applied to meta-analysis. ${ }^{64-66}$ Trial sequential analysis depends on the quantification of the required information size (the required sample size of the meta-analysis). In this context, the smaller the required information size the more lenient the trial sequential monitoring boundaries are, and accordingly, the more lenient the criteria for statistical significance will be. On the basis of predetermined criteria $^{53}$ we calculated the diversity-adjusted required information size based on the diversity $\left(\mathrm{D}^{2}\right)$ among the included trials. ${ }^{62}$ We conducted the trial sequential analyses with an intention to maintain an overall $5 \%$ risk of a type I error, which is the standard in most meta-analyses and systematic reviews. We calculated the required information size to detect or reject an intervention effect of a $10 \%$ relative risk reduction with a risk of a type II error of $20 \%$ (power of $80 \%$ ). For the dichotomous outcomes, the event proportion in the control group was based on the data from the meta-analysis. For the continuous outcomes, we calculated the required information size to detect or reject the achieved differences from the meta-analyses. We used TSAV.0.9 $\beta$ for these analyses. ${ }^{67}$

\section{RESULTS}

Figure 1 summarises the result of the search. We excluded 58 references after further evaluation. The reason for exclusion was a lack of predefined differences in glycaemic targets (36 trials); participants were not patients with type 1 diabetes mellitus or we could not separate data on patients with type 1 diabetes mellitus (10 trials); the trial was not randomised (11 studies); or the trial included only pregnant participants ( 1 trial). Fourteen references were not identified in the original search, but were retrieved from additional sources, of whom only one was a randomised clinical trial. ${ }^{68}$ Excluded studies are listed in web appendix 3.

We included 18 randomised trials, described in 136 publications. All trials were published in English. The trials included 2254 participants, of whom 1110 were randomised to target intensive glycaemic control versus 1144 to conventional glycaemic control. Table 1 shows key characteristics of the included trials and table 2 shows key characteristics of the trial participants.

The intervention target for glycaemic control varied among trials in both the intensive and conventional groups (table 3). Some trials predefined the intensive glycaemic target in terms of $\mathrm{HbA}_{1 \mathrm{c}}$ or preprandial or postprandial blood glucose concentration (table 3). Trials intending to lower the blood glucose in the intervention group to a larger extent than in the conventional group were included. In contrast, trials investigating whether glycaemia were lowered more by one treatment than another, for example, by different insulin regimens but without specifying differences in glucose targets or intentions with respect to differences in glucose levels, were excluded. Achieved treatment targets varied among trials, in general, the achieved $\mathrm{HbA}_{1 \mathrm{c}}$ seldom reached the planned target.

A trial by Linn et al $2003^{69}$ was never published. Through correspondence, it was stated that no publication was made due to lack of statistical significance.

We used the author's definitions of type 1 diabetes mellitus. Seven trials reported the diagnostic criteria for type 1 diabetes mellitus. These trials included patients with C-peptide level $<0.1-0.2 \mathrm{pmol} / \mathrm{mL}$ in the postabsorptive state and/or $6 \mathrm{~min}$ after intravenous injection of $1 \mathrm{mg}$ glucagon. ${ }^{3-46}{ }^{69-86}$ All trials excluded participants with severe concurrent illnesses, except for The Minnesota DCCT $^{83-86}$ which had kidney transplantation as an inclusion criterion. 
The DCCT ${ }^{3-46}$ contributed with most of the participants $(63.9 \%)$. The trial intervention period had a mean of 6.5 years, and was stopped 1 year before originally planned due to the positive results (especially on microvascular complications) in the intensive intervention group. EDIC is the observational long-term follow-up study including $96 \%$ of the living patients from DCCT. In the EDIC, patients were no longer randomised to different glycaemic targets, but were still seen regularly at the clinics, and patients in the former conventional group were offered intensive glycaemic control (similar to the former intensive group). A trial design with an intervention period and an epidemiological follow-up period were seen in seven of the included trials.

Owing to the relatively short intervention time in the included trials (excluding the DCCT) and young participants, the event-rate was relatively low for most of the outcomes. We therefore performed a Peto OR and continuity adjustment ad modum Sweeting for zero events with a value of 0.01 , to test whether it would change the statistical significance for the following outcomes: allcause mortality, cardiovascular mortality, retinopathy, retinal photocoagulation, nephropathy, end-stage renal disease and hypoglycaemia. The sensitivity analyses did not change the results noticeably for any of the outcomes.

\section{Bias risk assessment}

The quality of the included trials was in general low, and all trials had a high risk of bias (table 4). Seven of the included trials had a low risk of bias according to generation of the sequence, and two trials had a low risk of bias according to allocation concealment (table 4). Only the DCCT ${ }^{3-46}$ was judged as a trial with lower risk of bias according to our definition, but still had high risk of bias due to incomplete data on outcomes and academic bias.

\section{Clinical outcomes}

All-cause mortality

Nine trials provided information on all-cause mortality in a total of 1971 participants, reporting 42 deaths (figure 2A). Meta-analysis showed no statistically significant effect of targeting intensive glycaemic control compared with conventional glycaemic control (risk ratio (RR) $1.16,95 \%$ CI 0.65 to $2.08 ; \mathrm{p}=0.61$ ). Heterogeneity was absent $\left(\mathrm{I}^{2}=0 \% ; \mathrm{p}=0.89\right)$. Sensitivity analysis including only data from intervention periods did not change the lack of statistical significance of the effect estimate (RR $1.16,95 \%$ CI 0.60 to 2.26; $\mathrm{p}=0.66$; figure $2 \mathrm{~B}$ ). Data from DCCT $^{3-46}$ were only available from the end of the intervention period (mean 6.5 years).

Worst-best case scenario showed statistical significance in favour of a conventional glycaemic target (RR 2.96, 95\% CI 1.86 to $4.71 ; \mathrm{p}<0.0001)$. Best-worst case scenario showed statistical significance in favour of an intensive glycaemic target (RR $0.45,95 \%$ CI 0.28 to 0.71 ; $\mathrm{p}=0.0007$ ).

The predefined subgroup analyses according to insulin types, risk of bias and publication status could not be performed due to lack of data. Subgroup analyses of the trials including participants at all ages versus trials only including participants over 18 years showed no statistically significant differences between subgroups, that is, no statistically significant interaction. Sensitivity
Figure 1 Flow diagram of identification of randomised clinical trials for inclusion.

Potentially relevant references identified from
database search $(n=3025)$ and additional sources $(n=14)$

References could clearly be excluded on the basis of title/abstract $(n=2845)$

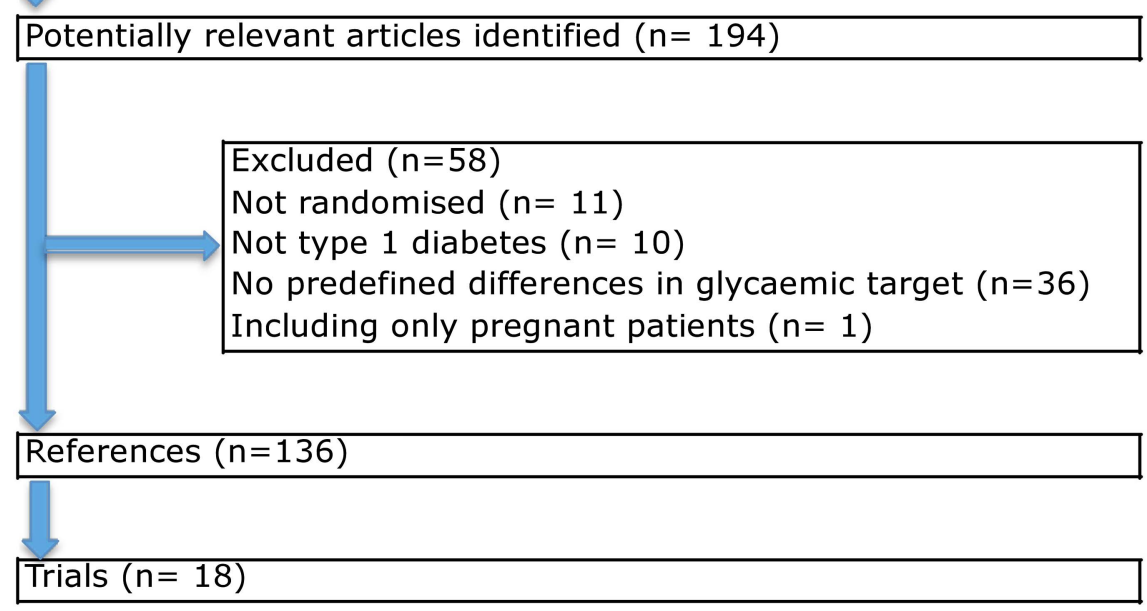


Table 1 Key characteristics of the included randomised clinical trials

\begin{tabular}{|c|c|c|c|}
\hline Trial & Location & Duration of intervention & Length of follow up \\
\hline DCCT/EDIC 1983 $3-46$ & 29 centres; USA and Canada & 6.5 years & 25 years \\
\hline Franklin et al ${ }^{104}$ & Scotland & 1 year & 1 year \\
\hline Hvidovre $1982^{68}$ & 1 centre; Denmark & 10 days & 180 days \\
\hline Kroc $1984^{112-117}$ & 6 centres; North America and England & 2 years* & 2 years \\
\hline Linn et $a l^{110}$ & Germany & 5 years & 5 years \\
\hline Linn et $a t^{69}$ & 4 centres; Germany & 3 years & 3 years at least $†$ \\
\hline Microalbuminuria 5051 & 9 centres; England and Wales & 5 years & 5 years \\
\hline Minnesota DCCT $1983^{83-86}$ & 2 centres; USA & 5 years & 5 years \\
\hline Oslo $1986^{73-80}$ & Norway & 4 years & 8 years \\
\hline Oxford $1983^{111}$ & England & 2 years & 2 years \\
\hline Perlman et al ${ }^{105}$ & 1 centre; Canada & 1 year & 1 year \\
\hline Service et $a^{\beta 1}$ & USA & 2.5 years & 2.5 years \\
\hline Shah et al ${ }^{101}$ & USA & $\begin{array}{l}\text { I: } 14 \text { days } \\
\text { C: } 1 \text { year }\end{array}$ & 1 year \\
\hline Steno 1a $1982^{72} 119120121$ & 1 centre; Denmark & 2 years & 8 years \\
\hline Steno $1 \mathrm{~b} 1986^{70-72}$ & 1 centre; Denmark & 2 years & 5 years \\
\hline Stockholm $1985^{87-98}$ & Sweden & 5 years & 14 years \\
\hline Verrillo et $a^{\beta 2}$ & Italy & 5 years & 5 years \\
\hline Hershey et $a l^{102}$ and White ${ }^{103}$ & 2 centres; USA & 1.5 years & 1.5 years \\
\hline \multicolumn{4}{|c|}{$\begin{array}{l}\text { *The study was planned to last for } 8 \text { months, but after the } 8 \text { months } 23 \text { participants (out of } 34 \text { ) in the intervention group and } 24 \text { participants } \\
\text { (out of } 34 \text { ) in the control group agreed to continue their intervention for additional } 16 \text { months, and all participants were re-evaluated after } \\
2 \text { years. } \\
\text { tWe only have the study protocol. No results were published and the author was not able to pass any data to us. } \\
\text { DCCT/EDIC, Diabetes Control and Complication Trial/Epidemiology of Diabetes Interventions and Complications; Minnesota DCCT, } \\
\text { Minnesota Diabetes Control and Complication Trial. }\end{array}$} \\
\hline
\end{tabular}

analysis excluding the trial with the longest duration $\left(\mathrm{DCCT} / \mathrm{EDIC}^{3-46}\right)$ did not change the lack of statistical significance of the effect estimate (RR 1.03, 95\% CI 0.53 to $1.98 ; \mathrm{p}=0.93)$. The Minnesota $\mathrm{DCCT}^{83-86}$ included participants with renal transplantation; sensitivity analyses excluding this trial did not change the lack of statistical significance (RR $1.48,95 \%$ CI 0.71 to $3.10 ; \mathrm{p}=0.30$ ).

Trial sequential analysis showed that only $1.18 \%$ of the required information size to detect or reject a $10 \%$ relative risk reduction was accrued, and the trial sequential monitoring boundaries were not crossed (figure 2C).

\section{Cardiovascular mortality}

Seven trials provided information on cardiovascular mortality in a total of 1802 participants (figure 3A). Meta-analysis showed no statistical significance of targeting intensive glycaemic control compared with conventional glycaemic control (RR $0.49,95 \%$ CI 0.19 to 1.24 ; $\mathrm{p}=0.13)$. Heterogeneity was absent $\left(\mathrm{I}^{2}=0 \% ; \mathrm{p}=0.84\right)$. Worst-best case scenario showed no statistical significance (RR 1.31, 95\% CI 0.66 to 2.57). Best-worst case scenario showed statistical significance in favour of targeting intensive glycaemic control (RR 0.27, 95\% CI 0.12 to $0.62 ; \mathrm{p}=0.002)$. Sensitivity analysis including only data from intervention periods did not change the lack of statistical significance of the effect estimate (RR 0.81, $95 \%$ CI 0.16 to 4.19 ; $\mathrm{p}=0.81$; figure $3 \mathrm{~B}$ ).

The predefined subgroups comparing insulin types, risk of bias and unpublished trials could not be performed, due to a lack of data. Subgroup analyses of the trials according to the age of participants showed no statistically significant differences between subgroups (ie, no significant interaction). Sensitivity analysis excluding the trial with the longest duration (DCCT/EDIC ${ }^{3-46}$ ) did not change the statistical significance of the effect estimate (RR $0.70,95 \%$ CI 0.19 to $2.66 ; \mathrm{p}=0.60$ ).

Trial sequential analysis showed that only $0.84 \%$ of the required information size to detect or reject a $10 \%$ relative risk reduction was accrued, and the trial sequential monitoring boundaries were not crossed (see web appendix 3, figure 9).

\section{Macrovascular complications}

Three trials provided information on macrovascular complications in a total of 1577 participants (figure 4A). We used the authors' definitions of macrovascular complications, which varied among the included trials, see web appendix 4 . A total of 87 participants with a macrovascular complication were reported of whom 83 participants were from DCCT/EDIC. ${ }^{3-46}$ Meta-analysis showed a statistically significant effect of targeting intensive glycaemic control compared with conventional glycaemic control (RR 0.63, 95\% CI 0.41 to $0.96 ; \mathrm{p}=0.03$ ). Heterogeneity was absent $\left(\mathrm{I}^{2}=0 \% ; \mathrm{p}=0.65\right)$. It was not possible to perform a sensitivity analysis including only data from intervention periods due to a lack of data.

Sensitivity analysis excluding the trial with the longest duration (DCCT/EDIC ${ }^{3-46}$ ) changed the statistical significance of the effect estimate to no statistically significant effect (RR $0.99,95 \%$ CI 0.15 to $6.57 ; \mathrm{p}=0.99$ ). 
Table 2 Key characteristics of the trial participants

\begin{tabular}{|c|c|c|c|c|c|}
\hline Trial & $\begin{array}{l}\text { Number of } \\
\text { participants } \\
\text { Intensive/ } \\
\text { conventional (total) }\end{array}$ & $\begin{array}{l}\text { Age (years) } \\
\text { Intensive (SD)/ } \\
\text { conventional (SD) }\end{array}$ & $\begin{array}{l}\text { Duration of disease at } \\
\text { baseline (years)* } \\
\text { Intensive (SD)/conventional } \\
\text { (SD) }\end{array}$ & $\begin{array}{l}\text { Weight at baseline }(\mathbf{k g})^{*} \\
\text { Intensive (SD)/ } \\
\text { conventional (SD) }\end{array}$ & $\begin{array}{l}\text { BMI at baseline }\left(\mathrm{kg} / \mathrm{m}^{2}\right)^{\star} \\
\text { Intensive }(\mathrm{SD}) / \\
\text { conventional (SD) }\end{array}$ \\
\hline DCCT/EDIC $1983^{3-46}$ & $711 / 730(1441)$ & $27.1(7.1) / 26.5(7.1)$ & $6.0(4.2) / 5.7(4.1)$ & NR & $23.4(2.7) / 23.5(2.9)$ \\
\hline \multirow{2}{*}{ Franklin et al ${ }^{104}$} & $C_{1}: 28$ & $C_{1}: 12.7(10.5-14.8) \dagger$ & $C_{1}: 3.2(1.7-6.7) \dagger$ & & $C_{1}: 0.38(-0.44-0.83) \dagger$ \\
\hline & $\mathrm{C}_{2}: 33(92)$ & $C_{2}: 14.1(11.7-15.6) \dagger$ & $\mathrm{C}_{2}: 4.8(2.6-8.6) \dagger$ & & $\mathrm{C}_{2}: 0.13(-0.55-1.0) \dagger^{* *}$ \\
\hline Hvidovre $1982^{68}$ & $7 / 9(16)$ & $27(21-37) \dagger / 28(17-35) \dagger$ & Newly diagnosed & $\begin{array}{l}93 \%(80-104) \dagger / 87.5 \% \\
(75-104) \dagger \ddagger\end{array}$ & NR \\
\hline Linn et al $\S^{69}$ & NR & NR & NR & NR & NR \\
\hline Microalbuminuria $^{505}$ & $36 / 34(70)$ & $37(19-59) \dagger / 37(17-58) \dagger$ & $21(6-35) / 18(7-34)$ & NR & $26(18-40)+/ 26(19-34) \dagger$ \\
\hline $\begin{array}{l}\text { Minnesota DCCT } \\
1983^{83-86}\end{array}$ & $52 / 47$ (99) & $35(6) / 36(8)$ & $23(6) / 21$ (5) & NR & $28.2(8.2) / 26(4.3)$ \\
\hline \multirow{3}{*}{ Oslo $1986^{73-80}$} & $\mathrm{I}_{1}: 15$ & $l_{1}: 26(19-42) \dagger$ & $\mathrm{I}_{1}: 12.8(6.8-20.8) \dagger$ & $\mathrm{I}_{1}: 71.7(10)$ & NR \\
\hline & $\mathrm{I}_{2}: 15$ & $\mathrm{I}_{2}: 26(18-38) \dagger$ & $\mathrm{I}_{2}: 12.8(6.4-23.3) \dagger$ & $\mathrm{I}_{2}: 68.6(9.3)$ & \\
\hline & $/ 15(45)$ & $/ 26(18-36) \dagger$ & $/ 12.7(6.8-12.0) \dagger$ & / 71.7 (10.5) & \\
\hline Steno $1 \mathrm{~b} 1986^{70-72}$ & $18 / 18(36)$ & $32(18-48) \dagger / 29(18-47) \dagger$ & $15(10-26) \dagger / 15(5-26) \dagger$ & NR & NR \\
\hline Stockholm $1985^{87-98}$ & 48/54 (102) & $30.0(7.5) / 31.7(7.3)$ & $17.9(6.4) / 16.3(4.9)$ & NR & $22.6(1.9) / 22.8(2.5)$ \\
\hline Verrillo et $a^{\beta^{2}}$ & $22 / 22(44)$ & $37(9.8) / 38(9.0)$ & $19(5) / 2(6)$ & NR & $25.8(3.5) / 26.2(3.7)$ \\
\hline $\begin{array}{l}\text { Hershey et al }{ }^{102} \text { and } \\
\text { White }{ }^{103}\end{array}$ & $17 / 17$ (34) & $13.9(2.8) / 14.3(2.7)$ & Newly diagnosed & NR & NR \\
\hline \multicolumn{6}{|c|}{$\begin{array}{l}{ }^{*} \text { Mean or median. } \\
\text { †Range. } \\
\text { †Bodyweight \% of ideal. } \\
\text { \$We only have the study protocol. No results were published and the author was not able to pass any data to us. } \\
\text { \Weight loss (kg). }\end{array}$} \\
\hline
\end{tabular}


Table 3 Glycaemic control

\begin{tabular}{|c|c|c|c|c|c|}
\hline Trial & $\begin{array}{l}\text { HbA }_{1 c} \text { at } \\
\text { baseline }(\%)^{\star} \\
\text { Intensive (SD)/ } \\
\text { conventional } \\
\text { (SD) }\end{array}$ & $\begin{array}{l}\text { Fasting blood } \\
\text { glucose at baseline } \\
\text { (mmol/L)* } \dagger \\
\text { Intensive (SD)/ } \\
\text { conventional (SD) }\end{array}$ & $\begin{array}{l}\text { Treatment target: } \\
\text { intensive glycaemic control }\end{array}$ & $\begin{array}{l}\text { Treatment target: conventional } \\
\text { glycaemic control }\end{array}$ & $\begin{array}{l}\text { Achieved } \mathrm{HbA}_{1 \mathrm{c}}(\%)^{*} \\
\text { Intensive (SD)/ } \\
\text { conventional (SD) }\end{array}$ \\
\hline DCCT/EDIC $1983^{3-46}$ & $\begin{array}{l}9.1(1.6) / 9.1 \\
(1.6)\end{array}$ & $\begin{array}{l}12.99(4.6) / 12.79 \\
(4.4)\end{array}$ & $\begin{array}{l}\mathrm{HbA}_{1 \mathrm{c}} \text { between } 4.05-6.05 \% \text {. FBG } \\
\text { between } 3.88-6.05 \mathrm{mmol} / \mathrm{L} \text { and } \\
9.99 \mathrm{mmol} / \mathrm{L} 90-120 \text { minutes } \\
\text { post-prandial and } 3.60 \mathrm{mmol} / \mathrm{L} \text { or } \\
\text { above after } 3 \mathrm{am}\end{array}$ & $\begin{array}{l}\mathrm{HbA}_{1 \mathrm{c}}<13.11 \% \text { and absence of } \\
\text { symptoms of glycosuria, } \\
\text { hyperglycaemia and ketonuria }\end{array}$ & $7.9(1.1) / 8.0(1.0)$ \\
\hline Franklin et $a l^{104}$ & $\begin{array}{l}10.2(2.0) / \\
C_{1}: 10.2(1.6) \\
C_{2}: 9.8(1.8)\end{array}$ & NR & $\begin{array}{l}\text { Expected lower } \mathrm{HbA}_{1 \mathrm{c}} \text { compared to } \\
\text { the conventional treated group, due } \\
\text { to Intensive pump treatment } \neq\end{array}$ & Conventional treatment & $\begin{array}{l}9.2(2.2) / \\
C_{1}: 10.3(1.7) \\
C_{2}: 10.1(1.7)\end{array}$ \\
\hline Hvidovre $1982^{68}$ & NR & $\begin{array}{l}23.3(12.6-39.5) \\
\S / 18.1(14.0-25.8) \S\end{array}$ & Near normo-glycaemia & Conventional treatment & $6.4 \mathrm{mmol} / \mathrm{L} / 9.0 \mathrm{mmol} / \mathrm{L}$ \\
\hline Kroc $1984^{112-117}$ & $\begin{array}{l}10.3(2.4) / 10.1 \\
(1.8)\end{array}$ & $\begin{array}{l}10.98(3.3) / 10.39 \\
(3.0)\end{array}$ & $\begin{array}{l}\text { BG between } 3.1 \text { and } 6.4 \mathrm{mmol} / \mathrm{L} \\
\text { before meal, and }<8.9 \mathrm{mmol} / \mathrm{L} \\
90 \text { minutes after meal }\end{array}$ & $\begin{array}{l}\text { To avoid extreme hyperglycaemia, } \\
\text { ketosis and symptomatic } \\
\text { hypoglycaemia }\end{array}$ & $8.1(1.2) / 10.0(2.3)$ \\
\hline Linn et al ${ }^{110}$ & $\begin{array}{l}12.4(5.5) / 13.1 \\
(6.2)\end{array}$ & $9.4(5.6) / 9.1(2.7) \emptyset$ & $\begin{array}{l}\mathrm{HbA}_{1 \mathrm{c}}<6.5 \% \text {, with a preprandial BG } \\
<6.8 \mathrm{mmol} / \mathrm{L} \text { and postprandial } \mathrm{BG} \\
<10 \mathrm{mmol} / \mathrm{L}\end{array}$ & $\begin{array}{l}\text { Conventional treatment with } \\
\text { absence of symptoms attributable } \\
\text { to glycosuria, or frequent } \\
\text { hypoglycaemia }\end{array}$ & $6.6(1.6) / 8.0(2.8)$ \\
\hline Linn et $a{ }^{\star \star 69}$ & NR & NR & Optimal glycaemic control & Conventional glycaemic control & NR \\
\hline Microalbuminuria 5051 & $\begin{array}{l}10.3(1.99) / 9.8 \\
(1.64)\end{array}$ & NR & $\begin{array}{l}\mathrm{HbA}_{1 \mathrm{c}} \leq 7.5 \% . \text { FBG between } \\
4-7 \mathrm{mmol} / \mathrm{L} \text { and a } 2 \text { hs postprandial } \\
\mathrm{BG} \leq 10 \mathrm{mmol} / \mathrm{L}\end{array}$ & Conventional treatment & $8.9(1.5) / 9.8$ \\
\hline $\begin{array}{l}\text { Minnesota DCCT } \\
1983^{83-86}\end{array}$ & NR & $\begin{array}{l}13.65(3.1) / 13.37 \\
(2.7)\end{array}$ & $\begin{array}{l}\mathrm{HbA}_{1 \mathrm{c}}<7 \% \text {, home capillary } \mathrm{BG} \\
\text { between } 3.33-8.32 \mathrm{mmol} / \mathrm{L} \text { before } \\
\text { meals and at bedtime, and } \\
\text { postprandial } \leq 9.98 \mathrm{mmol} / \mathrm{L}\end{array}$ & $\begin{array}{l}\text { To avoid hyperglycaemic } \\
\text { symptoms with } 60 \% \text { of home } \\
\text { capillary } \mathrm{BG}>11.10 \mathrm{mmol} / \mathrm{L} \text { and } \\
20 \%>16.65 \mathrm{mmol} / \mathrm{L} \text {. Since } 1983 \\
\text { target changed to } \mathrm{HbA}_{1 \mathrm{c}} \mathrm{A} 1<12 \%\end{array}$ & $9.6(1.6) / 11.7(0.13)$ \\
\hline Oslo $1986^{73-80}$ & $\begin{array}{l}\mathrm{I}_{1}: 9.4(1.5) \\
\mathrm{I}_{2}: 10.1(1.9) \\
/ 9.5(1.9)\end{array}$ & $\begin{array}{l}\mathrm{I}_{1}: 8.1(1.0) \\
\mathrm{I}_{2}: 9.1(0.9) \\
/ 8.2(1.0)\end{array}$ & Near normo-glycaemia & Conventional treatment & $\begin{array}{l}\mathrm{I}_{1}: 9.4(1.5) \\
\mathrm{I}_{2}: 10.1(1.9) \\
/ 9.5(1.9)\end{array}$ \\
\hline Oxford $1983^{111}$ & $\begin{array}{l}11.7(1.6) / 11.8 \\
(2.1)\end{array}$ & NR & Preprandial BG between 4-7 mmol/1 & Conventional treatment & $9.5(1.6) / 10.33(2.31)$ \\
\hline Perlman et $a l^{105}$ & $\begin{array}{l}14.6(2.4) / 15.8 \\
(4.8)\end{array}$ & $\begin{array}{l}28.4(14.4) / 25.8 \\
(8.9) \dagger \dagger\end{array}$ & $\begin{array}{l}\text { Fasting euglycaemia and normal } \\
\text { glucose excursions }\end{array}$ & $\begin{array}{l}\text { Preprandial BG between } 4.4- \\
10.0 \mathrm{mmol} / \mathrm{L} \text { and no glycosuria }\end{array}$ & $11.2(4.5) / 12.9(2.9)$ \\
\hline Service et $a{ }^{\beta 1}$ & $10.4 / 9.6$ & $8.9 / 9.2$ & $\begin{array}{l}\mathrm{HbA}_{1 \mathrm{c}} \text { within normal range and mean } \\
\text { plasma glucose }<11.1 \mathrm{mmol} / \mathrm{L}\end{array}$ & $\begin{array}{l}\text { Conventional treatment to eliminate } \\
\text { symptoms with a mean plasma } \\
\text { glucose }<11.1 \mathrm{mmol} / \mathrm{L}\end{array}$ & $9.8 / 9.6$ \\
\hline Shah et al $\left.\right|^{101}$ & $\begin{array}{l}18.2(6.2) / 15.9 \\
(5.6)\end{array}$ & NR & $\begin{array}{l}\text { BG between } 3.3-4.4 \mathrm{mmol} / \mathrm{L} \\
\text { preprandial and } 1 \mathrm{~h} \text { postprandial }\end{array}$ & $\begin{array}{l}\mathrm{BG}<7.8 \mathrm{mmol} / \mathrm{L} \text { preprandial and } \\
11.1 \mathrm{mmol} / \mathrm{L} \text { after } 1 \mathrm{~h} \text { postprandial }\end{array}$ & $7.2(2.4) / 10.8(4.5)$ \\
\hline
\end{tabular}


Table 3 Continued

\begin{tabular}{|c|c|c|c|c|c|}
\hline Trial & $\begin{array}{l}\mathrm{HbA}_{1 \mathrm{c}} \text { at } \\
\text { baseline (\%)* } \\
\text { Intensive (SD)/ } \\
\text { conventional } \\
\text { (SD) }\end{array}$ & $\begin{array}{l}\text { Fasting blood } \\
\text { glucose at baseline } \\
\text { (mmol/L)* } † \\
\text { Intensive (SD)/ } \\
\text { conventional (SD) }\end{array}$ & $\begin{array}{l}\text { Treatment target: } \\
\text { intensive glycaemic control }\end{array}$ & $\begin{array}{l}\text { Treatment target: conventional } \\
\text { glycaemic control }\end{array}$ & $\begin{array}{l}\text { Achieved } \mathrm{HbA}_{1 \mathrm{c}}(\%)^{\star} \\
\text { Intensive (SD)/ } \\
\text { conventional (SD) }\end{array}$ \\
\hline $\begin{array}{l}\text { Steno 1a } 1982^{72119-} \\
121\end{array}$ & $\begin{array}{l}9.6(1.6) / 8.8 \\
(1.4)\end{array}$ & $9.6 / 8.8$ & $\begin{array}{l}\text { Postprandial } B G<9 \mathrm{mmol} / \mathrm{L} \text { and } \mathrm{no} \\
\text { glucosuria }\end{array}$ & $\begin{array}{l}\text { Postprandial } \mathrm{BG}<15 \mathrm{mmol} / \mathrm{L} \text { and } \\
24 \mathrm{~h} \text { glucose excretion }<20 \mathrm{~g} \text {. After } \\
1 \text { year restriction was added about } \\
\text { no hypoglycaemia or ketonuria }\end{array}$ & $7.6(0.9) / 8.1(1.1)$ \\
\hline Steno $1 \mathrm{~b} 1986^{70-72}$ & $\begin{array}{l}9.5(6.6-13.6) \S / \\
9.3(7.0-11.7) \S\end{array}$ & $10.0(8.5) / 11.3(8.5)$ & $\begin{array}{l}\text { Fasting blood glucose between } \\
4-7 \mathrm{mmol} / \mathrm{L} \text { and postprandial } \mathrm{BG} \\
\text { between } 5-10 \mathrm{mmol} / \mathrm{L} \text { and avoiding } \\
\text { of blood glucose }<3 \mathrm{mmol} / \mathrm{L}\end{array}$ & $\begin{array}{l}\text { Postprandial morning BG } \\
<15 \mathrm{mmol} / \mathrm{L} \text { and } 24 \mathrm{~h} \text { glucose } \\
\text { excretion }<20 \mathrm{~g}\end{array}$ & $7.9(1.1 / 9.1(1.2)$ \\
\hline Stockholm 1985 ${ }^{87-98}$ & $\begin{array}{l}9.5(0.2) / 9.4 \\
(0.2)\end{array}$ & NR & $\begin{array}{l}\text { Intensified treatment with individual } \\
\text { goals for each patient }\end{array}$ & $\begin{array}{l}\text { Conventional treatment by reduced } \\
\text { BG without giving rise to serious } \\
\text { hypoglycaemia }\end{array}$ & $\begin{array}{l}7.26(0.85) / 8.13 \\
(1.10)\end{array}$ \\
\hline Verrillo et $a^{\beta 2}$ & $\begin{array}{l}11.1(1.1) / 11.8 \\
(1.9)\end{array}$ & $11.1(1.1) / 11.8(1.9)$ & $\begin{array}{l}\text { Normo-glycaemia with absence for } \\
\text { hypoglycaemia and avoiding } B G \\
<3 \mathrm{mmol} / \mathrm{L} \text {. Preprandial } \mathrm{BG} \text { between } \\
4-8 \mathrm{mmol} / \mathrm{L} \text { and } 120 \text { minutes } \\
\text { postprandial } \mathrm{BG}<10 \mathrm{mmol} / \mathrm{L}\end{array}$ & $\begin{array}{l}\text { Preprandial morning } B G<12 \mathrm{mmol} / \mathrm{L} \\
\text { and } 24 \mathrm{~h} \text { urinary glucose excretion } \\
\text { under } 20 \mathrm{~g}\end{array}$ & $8.7(0.5) / 10.5(0.5)$ \\
\hline $\begin{array}{l}\text { Hershey et } \text { al }^{102} \text { and } \\
\text { White }^{103}\end{array}$ & $8.26 / 9.96$ & NR & $\begin{array}{l}\text { Preprandial BG between } \\
3.9-6.7 \mathrm{mmol} / \mathrm{L} \text {, and avoidance of } \\
\text { hyperglycaemia }\end{array}$ & $\begin{array}{l}\text { Preprandial BG between } \\
4.4-10.0 \mathrm{mmol} / \mathrm{L} \text {, and avoidance of } \\
\text { hyperglycaemia }\end{array}$ & NR \\
\hline
\end{tabular}

\section{*Mean or median.}

†Converted from $\mathrm{mg} / \mathrm{dL}$ to $\mathrm{mmol} / \mathrm{L}$ by dividing by 18 .

$\ddagger$ An aim for a lower glycaemic target in the intensive group compared to the conventional group was confirmed by the author.

§Range.

१Mean glucose level.

${ }^{*}$ We only have the study protocol. No results were published and the author was not able to pass any data to us.

††Random plasma glucose.

DCCT/EDIC, Diabetes Control and Complication Trial/Epidemiology of Diabetes Interventions and Complications; HbA 1 , glycated haemoglobin $\mathrm{A}_{1 \mathrm{c}}$; Minnesota DCCT, Minnesota Diabetes

Control and Complication Trial;NR, not reported; $I_{1}$, intensive group $1 ; I_{2}$, intensive group 2; $C_{1}$, conventional group $1 ; C_{2}$, conventional group 2 . FBG, fasting blood glucose; BG, blood

glucose. 
Trial sequential analysis showed that only $3.84 \%$ of the required information size to detect or reject a $10 \%$ relative risk reduction was accrued so far, and the trial sequential monitoring boundaries were not crossed (figure 4B).

\section{Nephropathy and end-stage renal disease}

Five trials provided information on nephropathy in a total of 1635 participants (figure 5A). The definition of nephropathy varied among the included trials from albumin excretions rate $>300 \mathrm{mg} / 24 \mathrm{~h}$ to a non-specific 'clinical nephropathy', see web appendix 4. Metaanalysis showed a statistically significant effect of targeting intensive glycaemic control compared with conventional glycaemic control (RR $0.37,95 \%$ CI 0.27 to $0.50 ; \mathrm{p}<0.00001)$. Heterogeneity was low $\left(\mathrm{I}^{2}=5 \%\right.$; $\mathrm{p}=0.38$ ). Sensitivity analysis including only data from intervention periods also found a statistical significance of the effect estimate (RR $0.35,95 \%$ CI 0.12 to 1.00 ; $\mathrm{p}=0.05$; figure $5 \mathrm{~B}$ ).

Trial sequential analysis showed that only $10.4 \%$ of the required information size to detect or reject a $10 \%$ relative risk reduction was accrued, and the trial sequential monitoring boundaries were not crossed (figure $5 \mathrm{C}$ ).

The effect of intensive glycaemic targets on end-stage renal disease was not statistically significant (RR 0.96, $95 \%$ CI 0.13 to $7.05,3$ trials, 124 participants). Sensitivity analysis including only data from intervention periods did not change the lack of statistical significance of the effect estimate (RR 1.02, 95\% CI 0.37 to 3.34). The Minnesota DCCT ${ }^{83-86}$ included participants with renal transplantation, and contributed with most data to the meta-analysis of this outcome. Sensitivity analyses excluding this trial did not change the lack of statistical significance (RR $0.59,95 \%$ CI 0.23 to 1.52 ).

\section{Severe adverse events}

Two trials provided information on severe adverse events in a total of 1515 participants (figure 6). A total of 56 participants with a severe adverse event were reported (all data were from intervention period) whereas 54 participants were from DCCT/EDIC. ${ }^{3-46}$ Meta-analysis showed no statistically significant effect of targeting intensive glycaemic control compared with conventional glycaemic control (RR 1.03, 95\% CI 0.61 to 1.72; $\mathrm{p}=0.92)$. Heterogeneity was absent $\left(\mathrm{I}^{2}=0 \% ; \mathrm{p}=0.98\right)$. Worst-best case scenario showed no statistical significance in favour of intensive glycaemic targets (RR 1.25, 95\% CI 0.76 to 2.04 ), and neither did best-worst case scenario (RR $0.73,95 \%$ CI 0.45 to 1.18 ). Subgroup analyses of the trials according to risk of bias, insulin type and the age of the participants could not be performed due to a lack of data.

Trial sequential analysis showed that only $1.89 \%$ of the required information size to detect or reject a $10 \%$ relative risk reduction was accrued, and the trial sequential monitoring boundaries were not crossed (see web appendix 3 , figure 10 ).

Table 4 Risk of bias of the included trials

\begin{tabular}{|c|c|c|c|c|c|c|c|}
\hline Trial & $\begin{array}{l}\text { Sequence } \\
\text { generation }\end{array}$ & $\begin{array}{l}\text { Allocation } \\
\text { concealment }\end{array}$ & Blinding & $\begin{array}{l}\text { Incomplete } \\
\text { outcome } \\
\text { data }\end{array}$ & $\begin{array}{l}\text { Selective } \\
\text { outcome } \\
\text { reporting }\end{array}$ & $\begin{array}{l}\text { Academic } \\
\text { bias }\end{array}$ & $\begin{array}{l}\text { Sponsor } \\
\text { bias }\end{array}$ \\
\hline DCCT/EDIC $1983^{3-46}$ & Adequate & Adequate & Adequate & Unclear & Adequate & Inadequate & Adequate \\
\hline Franklin et $a l^{104}$ & Adequate & Adequate & Inadequate & Adequate & Unclear & Adequate & Adequate \\
\hline Hvidovre $1982^{68}$ & Unclear & Unclear & Unclear & Adequate & Unclear & Adequate & Unclear \\
\hline Kroc $1984^{112-117}$ & Unclear & Unclear & Adequate & Adequate & Unclear & Adequate & Adequate \\
\hline Linn et al ${ }^{110}$ & Adequate & Unclear & Unclear & Unclear & Unclear & Adequate & Unclear \\
\hline Linn et $a l^{69}$ & Unclear & Unclear & Unclear & Unclear & Adequate & Inadequate & Unclear \\
\hline Microalbuminuria 5051 & Unclear & Unclear & Unclear & Adequate & Unclear & Adequate & Adequate \\
\hline $\begin{array}{l}\text { Minnesota DCCT } \\
1983^{83-86}\end{array}$ & Unclear & Unclear & Unclear & Adequate & Unclear & Adequate & Adequate \\
\hline Oslo $1986^{73-80}$ & Adequate & Unclear & Adequate & Unclear & Unclear & Adequate & Inadequate \\
\hline Oxford $1983^{111}$ & Unclear & Unclear & Adequate & Adequate & Unclear & Adequate & Adequate \\
\hline Perlman et al ${ }^{105}$ & Unclear & Unclear & Unclear & Adequate & Unclear & Adequate & Adequate \\
\hline Service et $a^{\beta 1}$ & Adequate & Unclear & Unclear & Unclear & Unclear & Adequate & Unclear \\
\hline Shah et $a l^{101}$ & Adequate & Unclear & Adequate & Adequate & Unclear & Adequate & Unclear \\
\hline $\begin{array}{l}\text { Steno 1a } 1982^{72} 119- \\
121\end{array}$ & Unclear & Unclear & Adequate & Unclear & Unclear & Adequate & Inadequate \\
\hline Steno $1 \mathrm{~b} 1986^{70-72}$ & Unclear & Unclear & Unclear & Adequate & Unclear & Inadequate & Inadequate \\
\hline Stockholm $1985^{87-98}$ & Adequate & Unclear & Adequate & Unclear & Unclear & Adequate & Inadequate \\
\hline Verrillo et $a \beta^{\beta 2}$ & Unclear & Unclear & Adequate & Unclear & Unclear & Adequate & Unclear \\
\hline $\begin{array}{l}\text { Hershey et } a l^{102} \text { and } \\
\text { White }^{103}\end{array}$ & Unclear & Unclear & Unclear & Adequate & Unclear & Adequate & Adequate \\
\hline
\end{tabular}




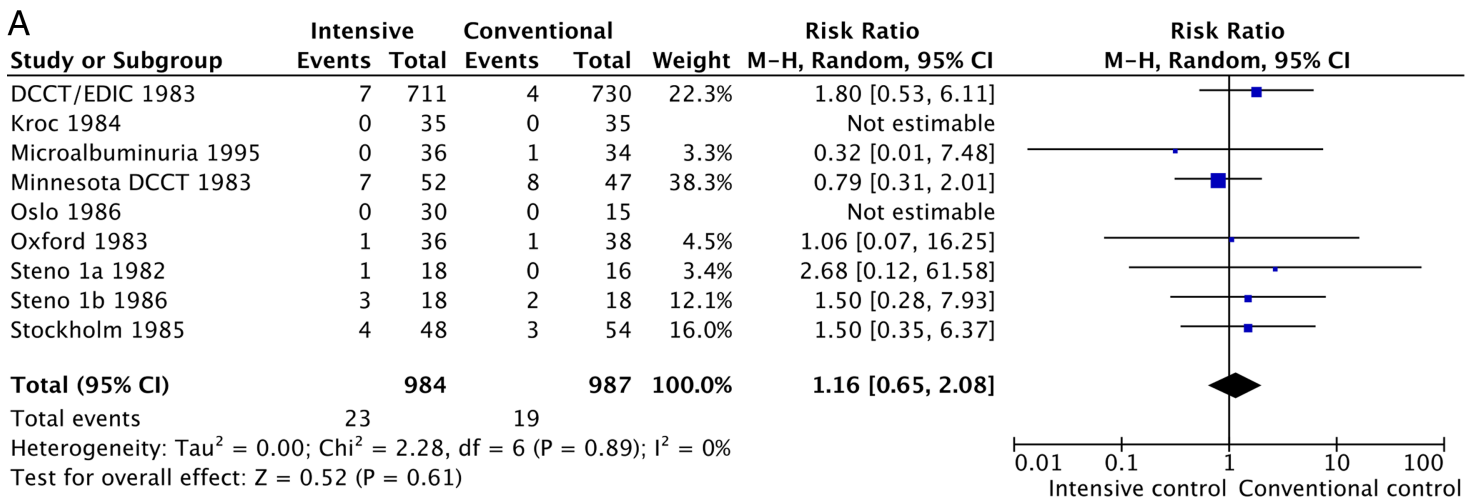

B

Intensive Conventional

Risk Ratio

Study or Subgroup Events Total Events

DCCT/EDIC 1983

$\begin{array}{rr}7 & 711 \\ 0 & 35 \\ 0 & 36\end{array}$

Kroc 1984

Microalbuminuria 1995

Minnesota DCCT 1983

$\begin{array}{ll}0 & 35 \\ 0 & 36\end{array}$

35
36

4

$\begin{array}{rr}\text { Total } & \text { Weight } \\ 730 & 29.5 \%\end{array}$

$29.5 \%$

35

$34 \quad 4.4 \%$

Oslo 1986

Oxford 1983

Stockholm 1985

752

52
30

1
8

$8 \quad 47 \quad 50.6 \%$

15

$50.6 \%$

$\begin{array}{lllll}1 & 36 & 1 & 38 & 5.9 \%\end{array}$

Random, 95\% Cl

Total $(95 \% \mathrm{Cl})$

948

$953100.0 \%$

Total events

19

15

Heterogeneity: $\mathrm{Tau}^{2}=0.00 ; \mathrm{Chi}^{2}=3.34, \mathrm{df}=4(\mathrm{P}=0.50) ; \mathrm{I}^{2}=0 \%$

Test for overall effect: $Z=0.44(P=0.66)$

$1.80[0.53,6.11]$

Not estimable

$0.32[0.01,7.48]$

$0.79[0.31,2.01]$

Not estimable

$1.06[0.07,16.25]$

$4.50[0.52,38.89]$

$1.16[0.60,2.26]$
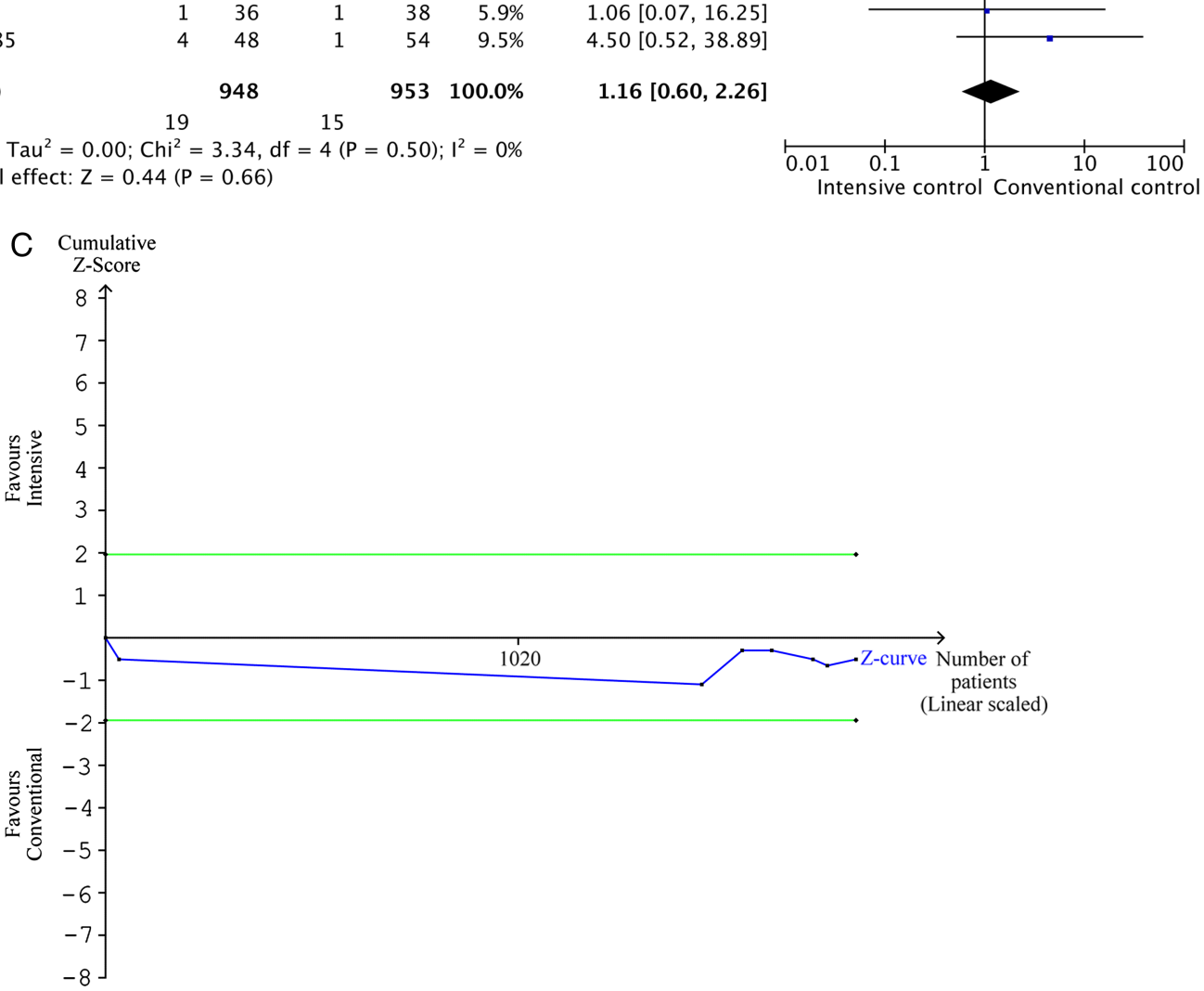

Figure 2 (A) Forest plot for all-cause mortality, meta-analysis of data to the longest follow-up. (B) Forest plot for all-cause mortality, meta-analysis of data to the end of the intervention period. (C) Trial sequential analysis of all-cause mortality. Trial sequence analysis revealed that only $1.18 \%(n=1971)$ of the diversity adjusted required information size of 167034 participants was accrued so far. The number was calculated based on a proportion of mortality of $1.9 \%$ in conventional glucose control group, a relative risk reduction of $10 \%$ in the intensive glycaemic group, $\alpha=5 \%, \beta=20 \%$, and $D^{2}=0 \%$. Solid blue line is the cumulative $z$-score, and it does not cross the horizontal solid green lines, illustrating the conventional level of statistical significance $(p=0.05)$. The cumulative $z$-score does not cross the trial sequential monitoring boundaries, which cannot be seen on the figure due to lack of data.

\section{Severe hypoglycaemia}

The definition of severe hypoglycaemia varied among trials from requiring third party assistance to requiring hospitalisation of the participants (see web appendix 4).
Eleven trials including 1983 participants provided information on severe hypoglycaemia (figure 7A). Meta-analysis showed a beneficial effect, that is, fewer events in favour of a conventional glycaemic target (RR 1.40, 95\% CI 1.01 to 


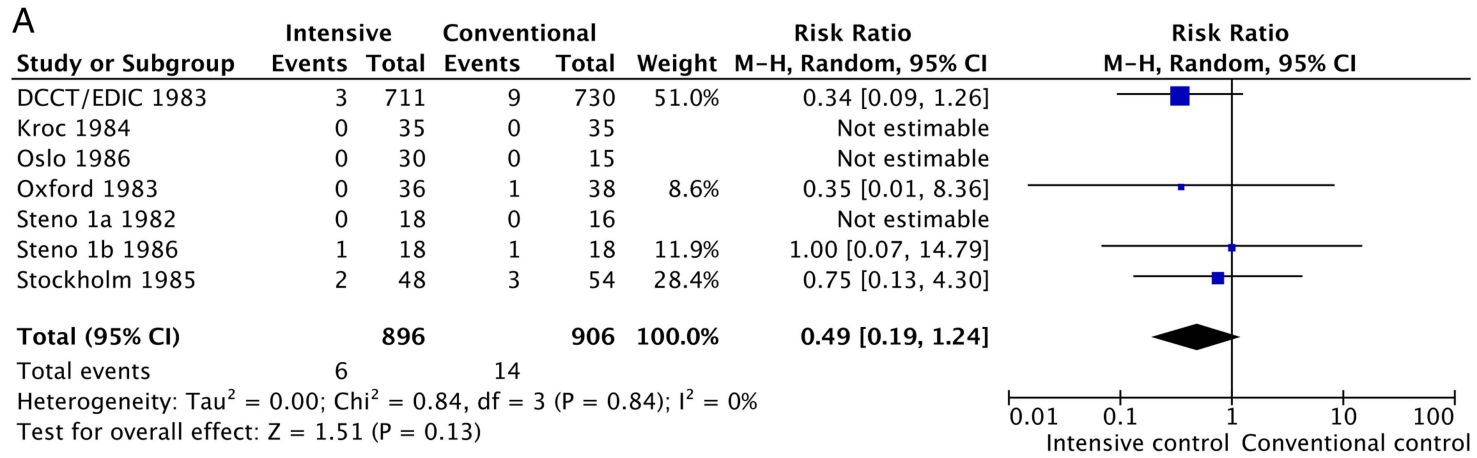

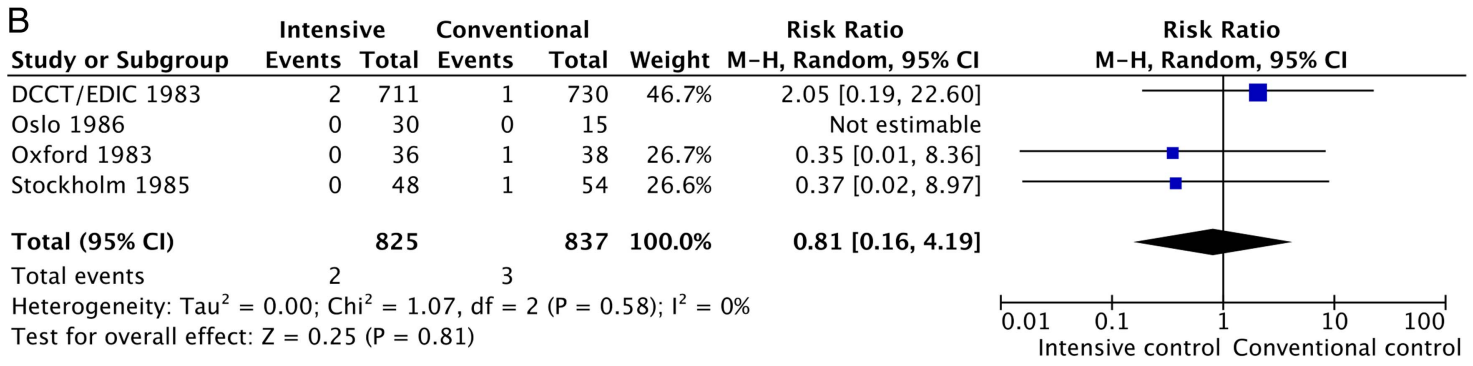

Figure 3 (A) Forest plot for cardiovascular mortality, meta-analysis of data to the longest follow-up. (B) Forest plot for cardiovascular mortality, meta-analysis of data to the end of the intervention period.

1.94; $\mathrm{p}=0.05)$. Heterogeneity was moderate $\left(\mathrm{I}^{2}=46 \%\right.$; $\mathrm{p}=0.05$ ). Sensitivity analysis including only data from intervention periods was not significantly affected in the random effects model (RR $1.35,95 \%$ CI 0.97 to 1.87 ; $\mathrm{p}=0.07$ ) but showed statistical significance in the fixed effect model favouring conventional glycaemic targets (RR $1.74,95 \%$ CI 1.57 to 1.93 ; $\mathrm{p}<0.00001$; figure $7 \mathrm{~B}$ ).

Trial sequential analysis showed that only $2.85 \%$ of the required information size to detect or reject a $10 \%$ relative risk reduction was accrued, and the trial sequential monitoring boundaries were not crossed (figure 7B).

\section{Body mass index}

Two trials provided information on body mass index in a total of 1276 participants (figure 8A). Meta-analysis of body mass index showed a statistically significant reduction to conventional glycaemic targets compared with intensive glycaemic targets (mean difference $1.13 \mathrm{~kg} / \mathrm{m}^{2}, 95 \%$ CI 0.18 to $2.07 ; \mathrm{p}=0.02)$. Heterogeneity was absent $\left(\mathrm{I}^{2}=0 \% ; \mathrm{p}=0.79\right)$. Only one trial $\left(\mathrm{DCCT}^{3-46}\right)$ reported BMI after the intervention period, and a sensitivity analysis including only data from intervention periods could not be performed.

Trial sequential analysis showed that $34.8 \%$ of the required information size to confirm the observed difference between the interventions was accrued, and the trial sequential monitoring boundaries were not crossed (figure 8B).

\section{Other assessed outcomes}

We found no statistically significant effect of targeting intensive glycaemic control on the incidence of cancer (RR 3.12, 95\% CI 0.33 to 29.65, 2 trials, 1515 participants) or non-fatal myocardial infarction (RR $0.58,95 \%$ CI 0.26 to $1.32,3$ trials, 1577 participants).

Ketoacidosis was not significantly affected in the random effects model (RR 1.44, 95\% CI 0.72 to $2.89,7$ trials, 1798 participants), but showed statistical significance in the fixed effect model favouring conventional glycaemic targets (RR 1.37, 95\% CI 1.01 to 1.86 ; $\mathrm{p}=0.04$ ). Sensitivity analysis including only data from intervention periods showed the same trend (see web appendix 3, figure $11 \mathrm{a}-\mathrm{c}$ ).

The definition of retinopathy varied among trials (see web appendix 4). Retinopathy was not significantly affected in the random effects model (RR $0.74,95 \%$ CI 0.50 to 1.10 , 9 trials, 1605 participants; web appendix 3, figure 12a,b), but showed statistical significance in the fixed effect model in favour of targeting intensive glycaemic control (RR 0.62, $95 \%$ CI 0.55 to $0.70 ; \mathrm{p}<0.00001)$. Heterogeneity was substantial $\left(I^{2}=62 \% ; p=0.007\right)$. Sensitivity analysis including only data from intervention periods showed the same trend (see web appendix 3, figure 12c).

Retinal photocoagulation was not significantly influenced in the random effects model (RR 0.41, 95\% CI 0.16 to 1.04 , 2 trials, 1238 participants; web appendix 3, figure 13a,b), but showed statistical significance in the fixed effect model favouring intensive glycaemic targets (RR $0.33,95 \%$ CI 0.23 to $0.48 ; \mathrm{p}<0.00001)$. Heterogeneity was moderate $\left(\mathrm{I}^{2}=48 \%\right.$; $\mathrm{p}=0.16)$. Only one trial $\left(\right.$ DCCT $\left.^{3-46}\right)$ reported retinal photocoagulation after the end of the intervention period, and a sensitivity analysis including only data from intervention periods could not be performed.

Sensitivity analyses excluding the trial with the longest duration (DCCT/EDIC ${ }^{3-46}$ ) changed the effect estimate 


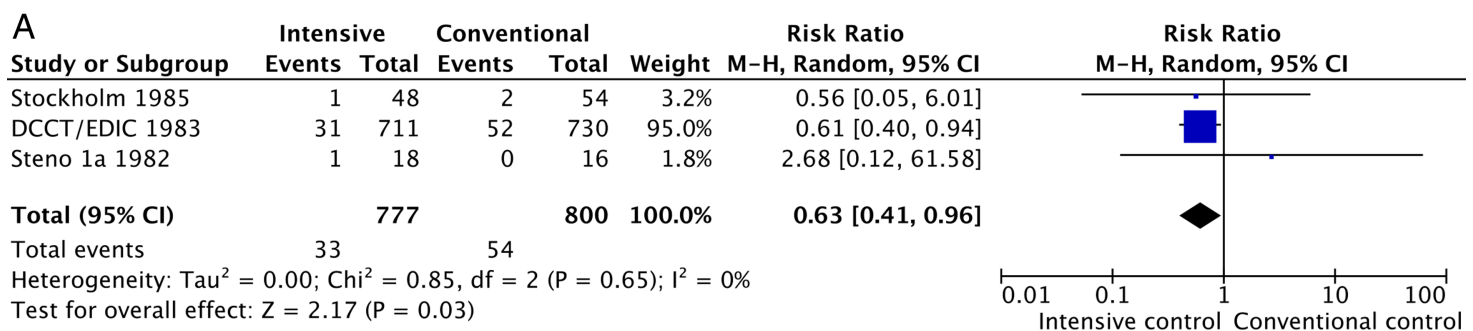

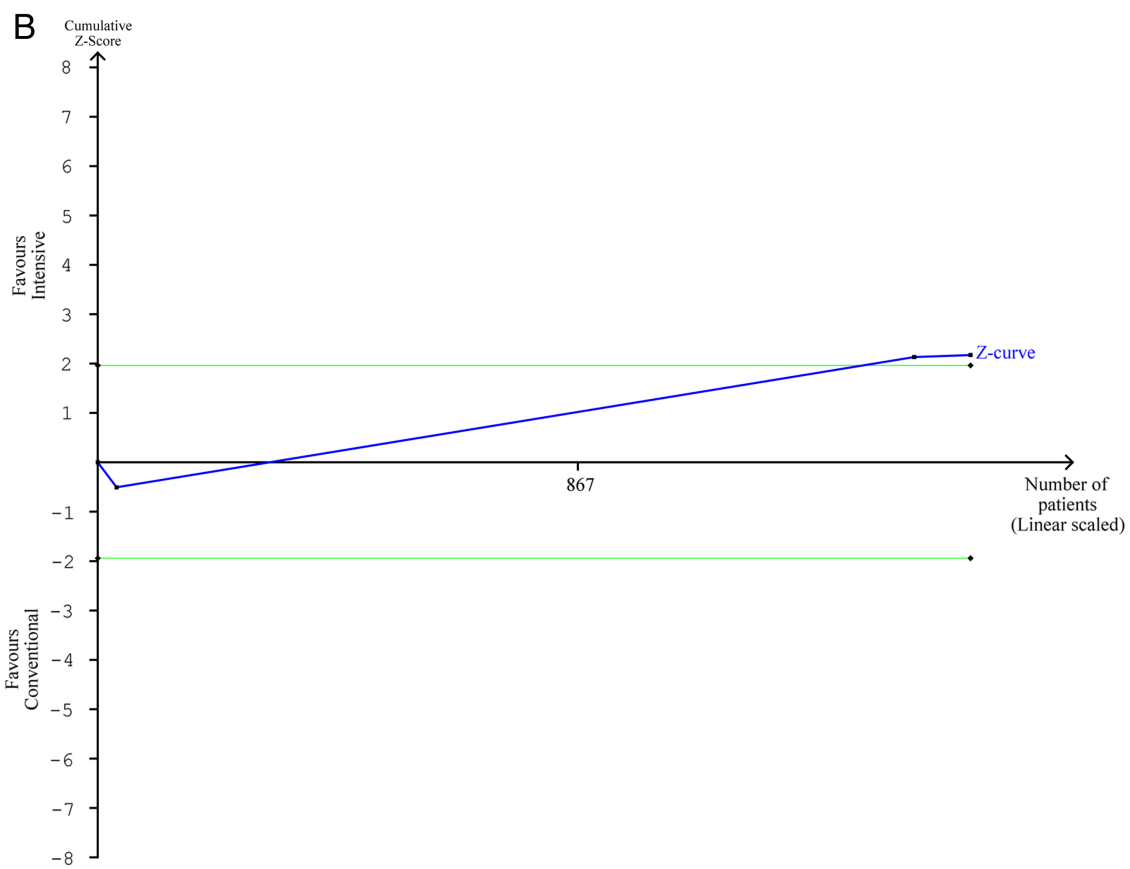

Figure 4 (A) Forest plot for macrovascular complications, meta-analysis of data to the longest follow-up. (B) Trial sequential analysis of macrovascular complications. Trial sequential analysis revealed that only $3.84 \%(n=1577)$ of the diversity adjusted required information size of 41068 participants was accrued so far. The number was calculated based on a proportion of macrovascular complications of $6.8 \%$ in conventional glucose control group, a relative risk reduction of $10 \%$ in the intensive glycaemic group, $\alpha=5 \%, \beta=20 \%$, and $D^{2}=0 \%$. Solid blue line is the cumulative $z$-score, and it crosses the horizontal solid green line, illustrating the conventional level of statistical significance $(p=0.05)$, favouring intensive glycaemic control. The cumulative $z$-score does not cross the trial sequential monitoring boundaries, which cannot be seen on the figure due to lack of data.

to a lack of statistical significance on the following outcomes: macrovascular complications, BMI, retinopathy and hypoglycaemia. Nephropathy was the only outcome that showed statistical significance before and after the sensitivity analysis excluding the trial with the longest duration (DCCT/EDIC ${ }^{3-46}$ ).

\section{Outcomes that could not be meta-analysed}

Congestive heart failure was only reported in DCCT $^{3-46}$ from the intervention period, and only one participant in each group developed heart failure. Non-fatal stroke was also only reported in DCCT $^{3-46}$ with zero events during the intervention period, but in the follow-up period one patient developed a non-fatal stroke in the intensive group and five patients in the conventionally treated group.

Amputation of lower extremity was only reported in DCCT/EDIC ${ }^{3-46}$ with seven patients in the intensive group and eight in the conventional group becoming amputated.
Revascularisation was only reported in DCCT/EDIC..$^{3-46}$ Peripheral revascularisation was reported with none patients in the intensive group and two in the conventional group. Coronary revascularisation was reported with 11 patients in the intensive group and 20 in the conventional group.

Health-related quality of life was assessed in two studies (Stockholm ${ }^{87-98}$ and DCCT $^{3-46}$ ), but only DCCT $^{3-46}$ published the results. Quality of life was measured with self-administered multiple choice 46-items specifically for the DCCT, ${ }^{3-46}$ but showed no statistically significant difference between the intensive and conventional groups.

Costs of intervention was only reported in DCCT, ${ }^{3-46}$ in which it was concluded that the intensive treatment (US\$4000/year) was three times as expensive as the conventional treatment (US $\$ 1700 /$ year), but may give cost savings as a result of the averted complications. 


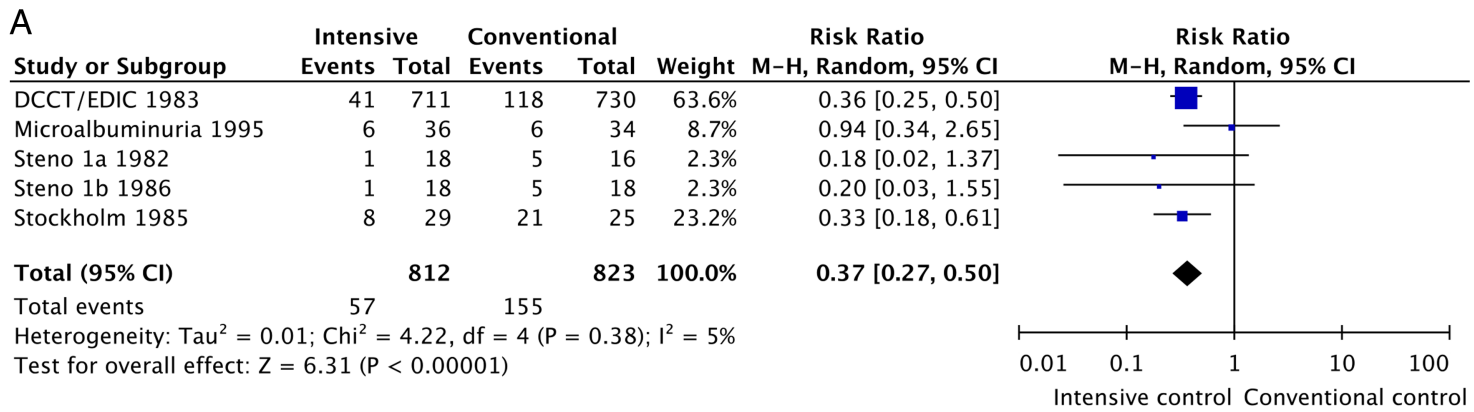

B

\begin{tabular}{|c|c|c|c|c|c|c|c|c|c|c|}
\hline Study or Subgroup & \multicolumn{2}{|c|}{ Intensive } & \multicolumn{2}{|c|}{ Conventional } & Weight & Risk Ratio & \multicolumn{4}{|c|}{$\begin{array}{c}\text { Risk Ratio } \\
\text { M-H, Random, } 95 \% \mathrm{Cl}\end{array}$} \\
\hline Microalbuminuria 1995 & 6 & 36 & 6 & 34 & $36.5 \%$ & $0.94[0.34,2.65]$ & & & & \\
\hline Stockholm 1985 & 0 & 48 & 8 & 54 & $11.1 \%$ & $0.07[0.00,1.11]$ & $\leftarrow$ & & & \\
\hline Total $(95 \% \mathrm{CI})$ & & 800 & & 829 & $100.0 \%$ & $0.35[0.12,1.00]$ & & & & \\
\hline
\end{tabular}

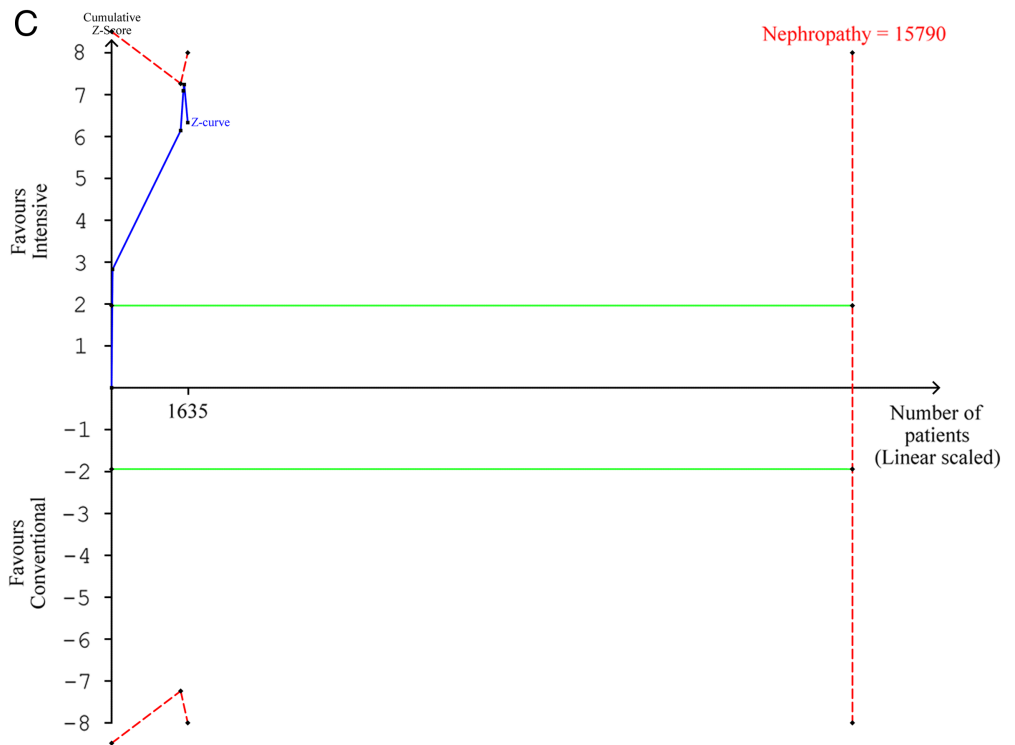

Figure 5 (A) Forest plot for nephropathy, meta-analysis of data to the longest follow-up. (B) Forest plot for nephropathy, meta-analysis of data to the end of the intervention period. (C) Trial sequential analysis of nephropathy. Trial sequential analysis revealed that only $10.4 \%(n=1635)$ of the diversity adjusted required information size of 15721 participants was accrued so far. The number was calculated based on a proportion of nephropathy of $18.8 \%$ in conventional glucose control group, a relative risk reduction of $10 \%$ in the intensive glycaemic group, $\alpha=5 \%, \beta=20 \%$, and $D^{2}=17 \%$. Solid blue line is the cumulative $z$-score, and it crosses the horizontal solid green line, illustrating conventional level of statistical significance $(p=0.05)$, favouring intensive glycaemic control. The cumulative z-score does touch the dotted red trial sequential monitoring boundaries after the second trial but returns to a level below the monitoring boundary hereafter.

Microvascular complications (as a composite outcome, using the authors' definition) were not reported for the included trials. Weight, adverse events, and mild hypoglycaemia were reported in a format that could not be included in a meta-analysis.

\section{DISCUSSION}

We identified 18 randomised clinical trials comparing the effects of targeting intensive glycaemic control versus conventional glycaemic control. Of these trials, only 12 ( $\mathrm{n}=2091$ participants) provided sufficient information to be included in one or more meta-analyses. None of the trials had low risk of bias on all bias domains, and only one trial $^{3-46}$ was considered to have lower risk of bias according to our adapted definition of lower risk of bias. Our key finding is that the risk of all-cause mortality and cardiovascular mortality was not statistically significantly different by targeting intensive versus conventional glycaemic control 


\begin{tabular}{|c|c|c|c|c|c|c|c|c|}
\hline \multirow[b]{2}{*}{ Study or Subgroup } & \multicolumn{2}{|c|}{ Intensive } & \multicolumn{2}{|c|}{ Conventional } & \multirow[b]{2}{*}{ Weight } & \multirow{2}{*}{$\begin{array}{c}\text { Risk Ratio } \\
\text { M-H, Random, } 95 \% \mathrm{Cl}\end{array}$} & \multirow{2}{*}{$\begin{array}{c}\text { Risk Ratio } \\
\mathrm{M}-\mathrm{H}, \text { Random, } 95 \% \mathrm{C} \\
\end{array}$} & \\
\hline & Events & Total & Events & Total & & & & \\
\hline DCCT/EDIC 1983 & 27 & 711 & 27 & 730 & $96.5 \%$ & $1.03[0.61,1.73]$ & & \\
\hline Oxford 1983 & 1 & 36 & 1 & 38 & $3.5 \%$ & $1.06[0.07,16.25]$ & & \\
\hline Total $(95 \% \mathrm{Cl})$ & & 747 & & 768 & $100.0 \%$ & $1.03[0.61,1.72]$ & & \\
\hline Total events & 28 & & 28 & & & & & \\
\hline $\begin{array}{l}\text { Heterogeneity: } \mathrm{Tau}^{2} \\
\text { Test for overall effec }\end{array}$ & $\begin{array}{l}0.00 ; C h \\
Z=0.10\end{array}$ & $\begin{array}{l}i^{2}=0 . \\
O(P=0\end{array}$ & $\begin{array}{l}.00, \mathrm{df}= \\
0.92)\end{array}$ & $P=0$ & $; 1$ & & $\begin{array}{ll}0.01 & 0.1 \\
\text { Intensive }\end{array}$ & 1 \\
\hline
\end{tabular}

Figure 6 Forest plot for severe adverse events, meta-analysis of data to the longest follow-up.

in patients with type 1 diabetes mellitus. However, targeting intensive glycaemic control might reduce the risk of macrovascular complications (assessed as a composite outcome), and nephropathy. However, analyses of only the intervention period could not be done for the composite macrovascular outcome, and data for nephropathy was based on surrogate markers. Consistent statistical significance could not be established for retinopathy, retinal photocoagulation or ketoacidosis. Importantly, meta-analyses of patient relevant outcomes were based on very sparse data, and the high risks of bias should be taken into account. No significant effects of targeting intensive glycaemic control were identified on the risks of severe adverse events, incidence of cancer, end-stage renal disease, non-fatal myocardial infarction or non-fatal stroke. The disadvantages of intensive glycaemic targets might be

\begin{tabular}{|c|c|c|c|c|c|c|c|c|}
\hline $\begin{array}{l}\text { A } \\
\text { Study or Subgroup }\end{array}$ & $\begin{array}{l}\text { Intens } \\
\text { Events }\end{array}$ & $\begin{array}{l}\text { ive } \\
\text { Total }\end{array}$ & $\begin{array}{l}\text { Convent } \\
\text { Events }\end{array}$ & $\begin{array}{l}\text { ional } \\
\text { Total }\end{array}$ & Weight & $\begin{array}{c}\text { Risk Ratio } \\
\text { M-H, Random, } 95 \% \mathrm{Cl}\end{array}$ & $\begin{array}{r}\text { Risk } \\
\text { M-H, Rand } \\
\end{array}$ & $\begin{array}{l}\text { Ratio } \\
\text { om, } 95 \% \mathrm{Cl}\end{array}$ \\
\hline DCCT/EDIC 1983 & 459 & 711 & 255 & 730 & $34.9 \%$ & $1.85[1.65,2.07]$ & & - \\
\hline Kroc 1984 & 6 & 35 & 1 & 35 & $2.4 \%$ & $6.00[0.76,47.29]$ & & \\
\hline Linn 1996 & 0 & 23 & 0 & 19 & & Not estimable & & \\
\hline Microalbuminuria 1995 & 5 & 36 & 5 & 34 & $6.7 \%$ & $0.94[0.30,2.98]$ & & \\
\hline Oslo 1986 & 8 & 30 & 7 & 15 & $11.4 \%$ & $0.57[0.26,1.28]$ & & \\
\hline Oxford 1983 & 1 & 36 & 1 & 38 & $1.4 \%$ & $1.06[0.07,16.25]$ & & \\
\hline Steno 1a 1982 & 3 & 18 & 2 & 16 & $3.5 \%$ & $1.33[0.25,7.00]$ & & \\
\hline Steno 1b 1986 & 5 & 18 & 5 & 18 & $7.6 \%$ & $1.00[0.35,2.87]$ & & 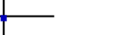 \\
\hline Stockholm 1985 & 34 & 48 & 29 & 54 & $27.6 \%$ & $1.32[0.97,1.79]$ & & $=$ \\
\hline Verillo 1988 & 2 & 22 & 1 & 22 & $1.9 \%$ & $2.00[0.20,20.49]$ & & \\
\hline White 1994 & 6 & 13 & 1 & 12 & $2.6 \%$ & $5.54[0.78,39.57]$ & & \\
\hline Total $(95 \% \mathrm{Cl})$ & & 990 & & 993 & $100.0 \%$ & $1.40[1.01,1.94]$ & & 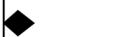 \\
\hline Total events & 529 & & 307 & & & & & \\
\hline $\begin{array}{l}\text { Heterogeneity: } \operatorname{Tau}^{2}=0 \\
\text { Test for overall effect: } Z\end{array}$ & $\begin{array}{l}\text { 8; } \mathrm{Chi}^{2}= \\
=1.99(\mathrm{P}\end{array}$ & $\begin{array}{l}=16.76 \\
=0.05\end{array}$ & 6, $d f=9$ & $P=0.05$ & $; 1^{2}=469$ & & $\begin{array}{cc}0.01 & 0.1 \\
\text { Intensive }\end{array}$ & 1 Conventic \\
\hline
\end{tabular}

B

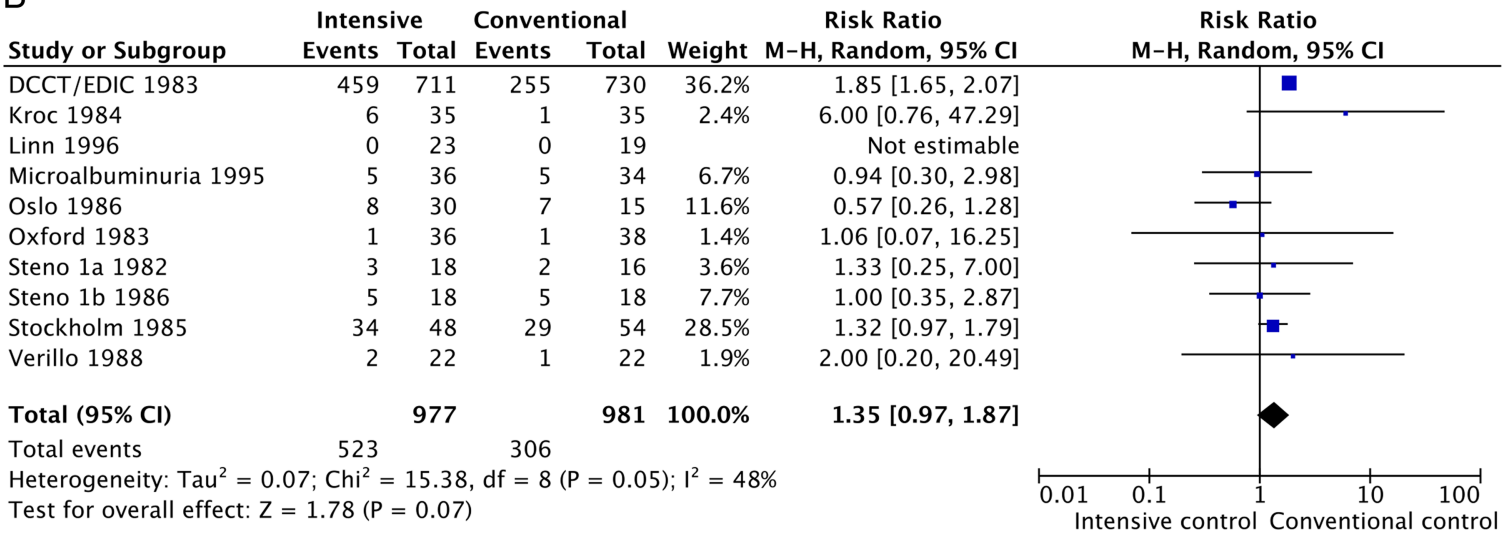

Figure 7 (A) Forest plot for severe hypoglycaemia, meta-analysis of data to the longest follow-up. (B) Forest plot for severe hypoglycaemia, meta-analysis of data to the end of the intervention period. (C) Trial sequential analysis of severe hypoglycaemia. Trial sequential analysis revealed that only $2.85 \%(n=1983)$ of the diversity adjusted required information size of 69579 participants was accrued so far. The number was calculated based on a proportion of severe hypoglycaemia of $30.9 \%$ in conventional glucose control group, a relative risk reduction of $10 \%$ in the intensive glycaemic group, $\alpha=5 \%, \beta=20 \%$, and $D^{2}=5 \%$. Solid blue line is the cumulative z-score, and it crosses the horizontal solid green lines, illustrating the conventional level of statistical significance $(p=0.05)$, favouring conventional glycaemic control. The cumulative $z$-score touch the traditional line of statistical significance but does not cross the trial sequential monitoring boundaries, which cannot be seen on the figure due to lack of data. 


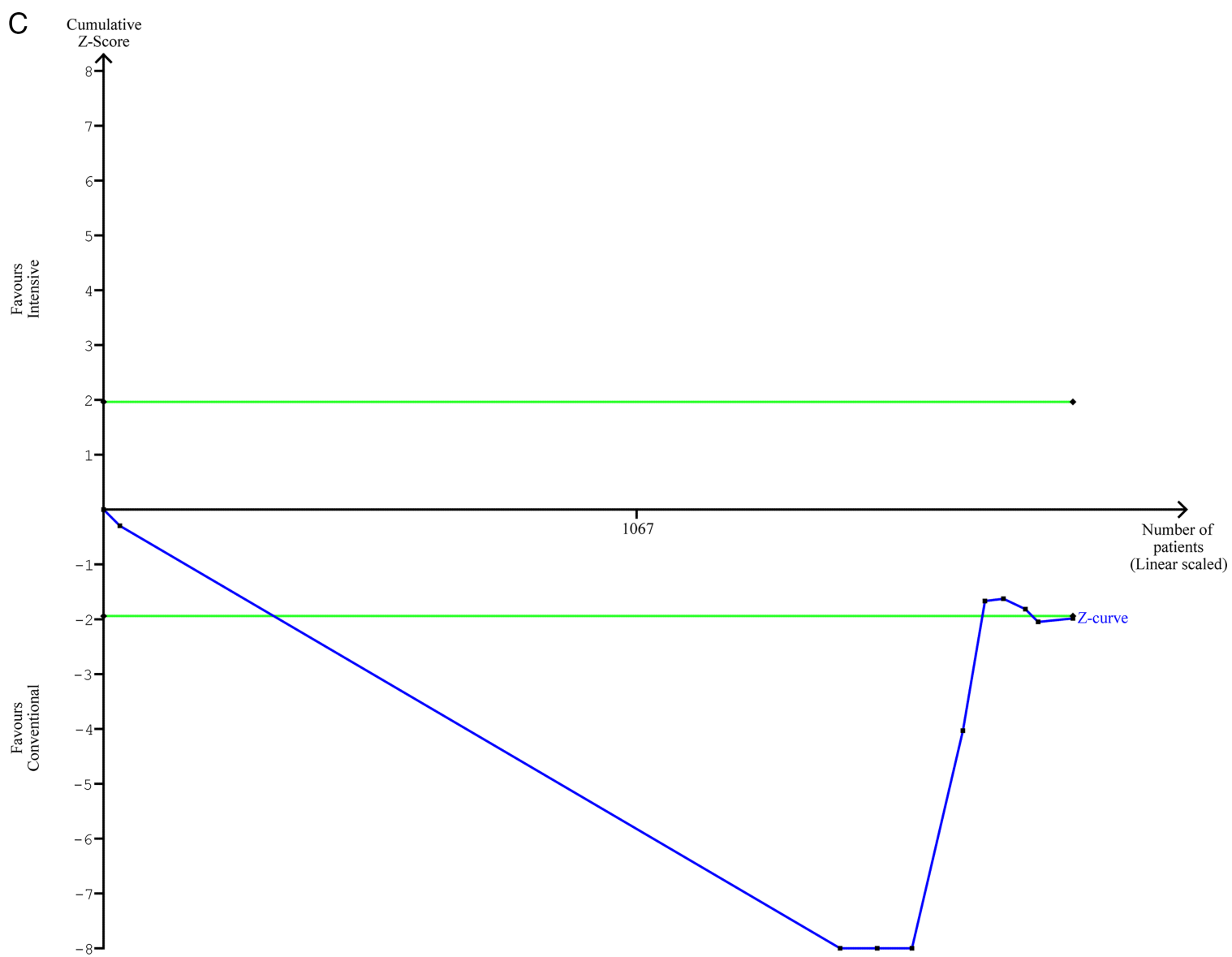

Figure 7 Continued

severe hypoglycaemia, ketoacidosis, and increase in body mass index, but data were sparse. Where available, analyses were made on data from the intervention period, and compared with data extracted to the longest follow-up; in general, these analyses showed no major differences.

After ended intervention, in the observational follow-up period, the patients were not required to continue the treatment into which they were randomised, but they were still included in the follow-up of their former group. This can lead to bias, but as long as the patients have had the intervention, they can provide data regarding the effect of the intervention. However, intensive glucose control is not applied as a temporary treatment, but as a lifelong intervention. The effects reported from observational periods of a trial following a temporary intervention may not be applicable to daily clinical practice and provide conclusive evidence of the consequences of continued use of the interventions beyond the initial intervention period.

The increased risk of ketoacidosis with intensive glycaemic targets may be due to different insulin administration in the two intervention groups. Many of the patients in intensive therapy were treated with insulin pumps, where the patients in the conventional group were treated with insulin injections. The insulin pumps from the 1980s and 1990s (where most of the trials were performed) are known to increase the risk of ketoacidosis, due to malfunction of the pump. The association between an intensive glycaemic target and increased risk of ketoacidosis may therefore be confounded by the mode of insulin administration.

\section{Strengths and limitations}

Our systematic review has several strengths. We based it on a published protocol $^{53}$ with rigid inclusion criteria for randomised clinical trials. We applied a comprehensive search with no language limitations or restrictions on outcomes reported in the trials. Two authors independently extracted data. We contacted corresponding authors of all trials for additional data and to clarify methodological details, but only a few authors responded. We tried to evaluate the strength of the available evidence with trial sequential analysis on all our primary and statistically significant secondary outcomes.

We have included trials with large differences in the patients' average duration of type 1 diabetes mellitus, length of the interventions, patients' age, and assessment of glycaemic control, as well as prespecified targets of glycaemic control. We included trials irrespective of the language of publication and outcomes reported.

The weaknesses of our analyses and conclusions mirror the weaknesses in the individual included trials, 

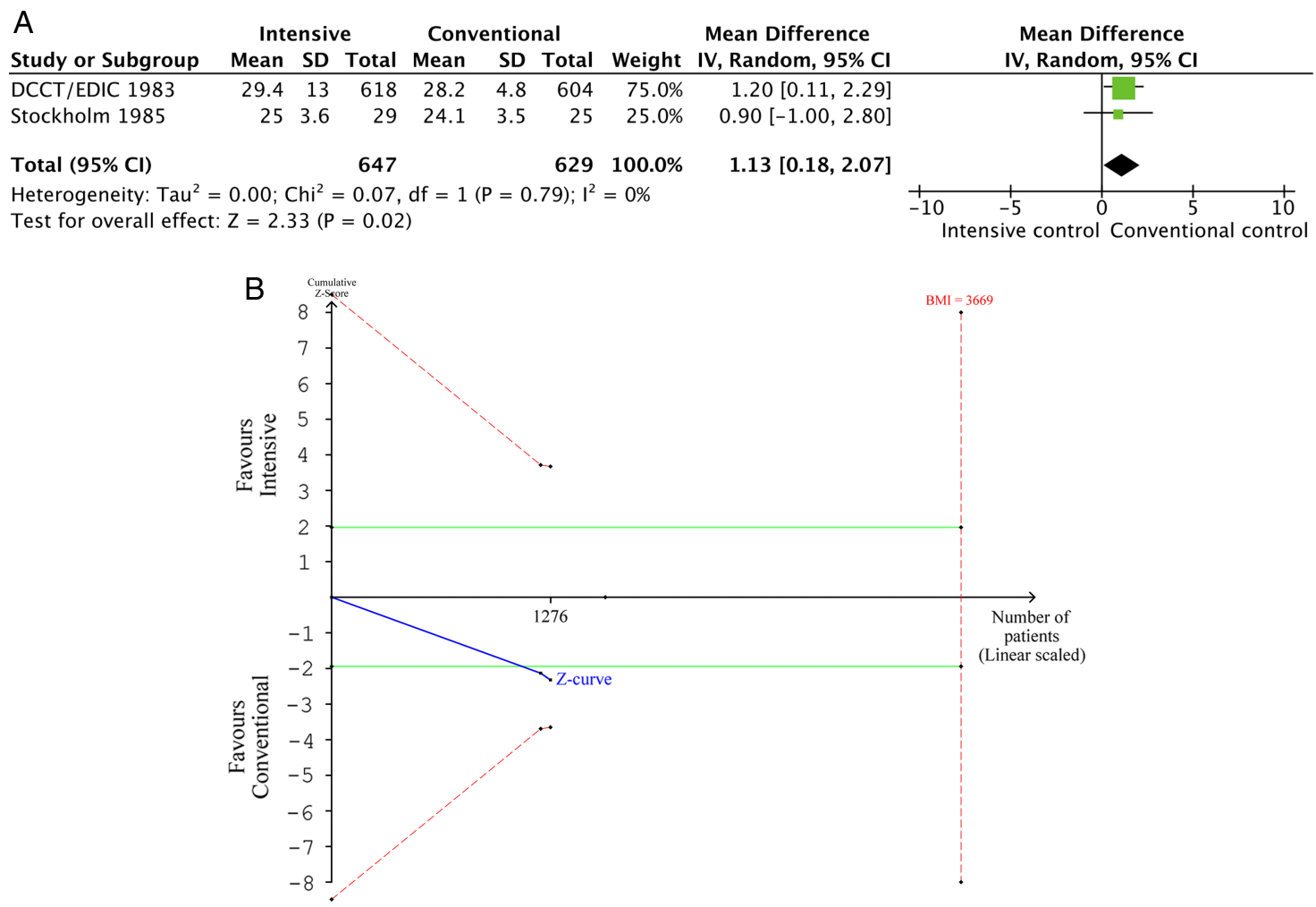

Figure 8 (A) Forest plot for body mass index, meta-analysis of data to the longest follow-up. (B) Trial sequential analysis of body mass index. Trial sequential analysis revealed that $34.8 \%(n=1276)$ of the diversity adjusted required information size of 3667 participants was accrued so far. The number was calculated based on a relative risk reduction of $10 \%$ in the intensive glycaemic group, $\alpha=5 \%, \beta=20 \%$, a mean difference of $1.13 \mathrm{~kg} / \mathrm{m}^{2}$ as achieved in the meta-analyses, and $D^{2}=0 \%$. Solid blue line is the cumulative $z$-score, and it crosses the horizontal solid green line, illustrating the conventional level of statistical significance $(p=0.05)$, favouring conventional glycaemic control. The cumulative $z$-score does not cross the dotted red trial sequential monitoring boundaries.

especially the high risk of bias, and the low number of patients included in the trials, highlighting the substantial lack of evidence on this topic. Only 1 of the 18 included trials was classified as lower risk of bias (DCCT $^{3-46}$ ) according to our definition of adequate sequence generation, allocation concealment and blinded outcome assessment. Such a definition does not rule out high risk of bias from other domains. ${ }^{52}$

We included a large range and thus a diverse group of patients with type 1 diabetes mellitus. However, due to a potential selection bias of for instance more healthy and motivated patients in clinical trials, it is difficult to evaluate exactly how typical the participants in each of the clinical trials may have been compared with the general population of patients with type 1 diabetes mellitus. Nevertheless, the heterogeneity in this review might indeed reflect the well-known heterogeneity in clinical practice. It is also important to remember that the patient-care applied in the intensive compared with the conventional groups is likely to be different from the everyday clinical practice, due to more frequent visits for the intensive groups and possible differences between the two settings in the awareness with respect to the blood glucose control.

We evaluated the strength of the available evidence by comprehensive analyses of the risk of sparse data and repetitive testing with trial sequential analysis. Even though the conventional meta-analyses of composite macrovascular complications, nephropathy and severe hypoglycaemia indicated a statistically significant effect estimate, trial sequential analysis showed that sufficient evidence to detect or reject a $10 \%$ relative risk reduction or risk increase was not available for any of these outcomes. This was also the case with the achieved difference in body mass index.

This meta-analysis is limited by an inability to use individual patient data to assess whether certain characteristics (eg, a history of cardiovascular events, degree of $\mathrm{HbA}_{1 \mathrm{c}}$ reduction and duration of disease at baseline) affect the degree of cardiovascular risk. We explored heterogeneity by sensitivity analyses and subgroup analyses at trial level.

Diagnostic criteria and definitions of outcomes varied among the trials and were not always well defined. This can potentially lower the validity of the results, but since the definition of an outcome does not change within the trial, it is not likely to substantially affect the results in favour of any of the groups, and it may still be used to see a trend. That is, unless a significant and undetected between-trial heterogeneity exists for the intervention effect, for example, outcomes incorrectly termed 
similarly, but representing different pathologies, in different trials.

In addition to the differences between the intensive glycaemic targets, the conventional target also varied among the trials. Furthermore, the measurement used to assess the levels of glycaemic control varied among the included trials. Some trials defined the glucose target by applying blood glucose, providing only a 'snapshot' of the overall glycaemic control. Most of the included trials expressed glycaemic targets as $\mathrm{HbA}_{1 \mathrm{c}}$, reflecting an average of the blood glucose concentration over several weeks. The treatment in the intensive treated group and the conventional treated group differed in many ways besides different glycaemic targets, including the number of doctor visits, insulin type and administration. For example, the intensive treated group in the DCCT $/ \mathrm{EDIC}^{3-46}$ could use insulin pumps or multiple daily injections (patients' and physicians' choice), whereas the conventional group had to use twice-daily insulin injections. No trial investigated the different glycaemic target keeping everything else the same. This is a major limitation, since it cannot be concluded that the effect we find between the groups is exclusively due to the use of different glycaemic targets.

The reporting of severe hypoglycaemia is problematic in several ways; first of all, the definitions of severe hypoglycaemia were diverse, some included incidents requiring a third party assistance and other hospitalisation. In addition, the design of the included trials made blinding of the participants difficult, which may lead to reporting bias. $^{52} 99100$ Many of the included trials were not designed or powered to assess our predefined outcomes, which explains the insufficient data from these trials. Furthermore, for some outcomes only a few trials could provide data. This increases the risk of outcome measure reporting bias. ${ }^{52}$

Four trials included only patients below the age of 18 years (Shah et $a l^{101}$ included adolescents age not defined).$^{101-105}$ These four trials did not report any macrovascular or microvascular outcomes, which might be due to the relatively short follow-up period. The effect of targeting intensive glycaemic control on patients younger than 18 years could therefore not be assessed.

\section{Relation to other studies}

Previous meta-analyses have been published. ${ }^{57-61} 106$ However, many of these claiming to assess the effect of targeting intensive glycaemic control versus conventional glycaemic control have included trials on the basis of achieved (ie, observed during follow-up) rather than targeted (ie, as randomly allocated) differences in glycaemic control. $^{58}$ For example, they included comparisons of insulin treatment regimens (continuous subcutaneous insulin infusions or multiple injections) with a similar target of $\mathrm{HbA}_{1 \mathrm{c}}$ below $6.5 \%$ in both intervention groups. ${ }^{107}$ This type of strategy for selection is potentially problematic, as the levels of glycaemic control targeted and achieved in a clinical trial represent different chosen variables. For example, unlike target levels, achieved levels of a variable cannot usually be guaranteed to have complete separation (at the patient level) between intervention groups. This precludes inferences with respect to causality between achieved levels of one outcome, for example, glucose control, and other outcomes, for example, vascular complications between groups. In contrast, target levels, if predefined to differ (ie, be completely separated) between groups, can better support inferences about causality. Therefore, to optimally assess the clinical effect of aiming for intensive glycaemic targets, which is probably a relevant question for the clinician as well as people trying to establish evidence-based guidelines, trials need to be meta-analysed on the basis of predefined differences in glycaemic targets.

Intensive glycaemic control is generally recommended in most countries due to the results of especially the DCCT/ EDIC. ${ }^{3-46}$ Only a few observational studies have assessed the effects of glycaemic levels and the outcomes we predefined to assess in patients with type 1 diabetes mellitus. However, we retrieved one observational study showing that improved glycaemic control was associated with lower risk of heart failure. ${ }^{108}$ However, such observational associations have well-known limitations as evidence. ${ }^{109}$

The so far largest meta-analysis on this topic by Fullerton et al included 10 of the 18 trials included in the present analysis. ${ }^{3-46} \quad 50 \quad 51 \quad 70-80 \quad 82-86 \quad 102 \quad 103 \quad 110-114$ They extracted the DCCT/EDIC ${ }^{3-46}$ as two separate trials: 'the primary prevention cohort' and 'the secondary intervention cohort' according to the presence of background retinopathy and microalbuminuria in the patients at baseline. They included one trial that did not meet standard criteria for randomisation. In contrast to Fullerton $e t a l^{106}$ we extracted DCCT as one trial (the primary and the secondary cohort together) and we included all trials that randomised patients to different targets, also if the target was not predefined but only an intention for better glycaemic control, and we did not have a time limit for the intervention period. In contrast to our results, Fullerton $e t$ al found an effect of intensive glycaemic control on retinopathy, but a sensitivity analysis showed that the effect was weaker regarding progression after manifestation. Consequently, the presence of retinopathy at treatment initiation could impact the relation between glucose-lowering and progression of retinopathy-a hypothesis that we did not specifically assess, but it should be considered in future trials and meta-analyses. A previous study from Stettler $e t a \tilde{l}^{7}$ also dealt exclusively with trials in which the patients were randomised to different glycaemic targets and included only 7 of 18 available trials. ${ }^{3-46} 5051 \quad 70-72818287-98111$

Another large meta-analysis by Egger $e t a \rho^{8}$ included 9 of the 18 trials included in the present systematic review. ${ }^{3-46} 4950$ 70-82 87-98 111-117 They also included five trials with different achieved $\mathrm{HbA}_{1 \mathrm{c}}$, but the patients were not randomised to different glycaemic targets. The differences in the setup and specification of the 
intervention of the relevant trials make such an approach prone to additional bias and therefore difficult to assess the benefits and harms of the intervention.

A newly published Cochrane review by Callaghan et $a l^{18}$ investigated the effect of intensive glycaemic control on neuropathy in patients with type 1 and 2 diabetes mellitus, and we therefore chose not to include neuropathy as an outcome.

Our systematic review and meta-analyses is largely dominated by the data from DCCT/EDIC, ${ }^{3-46}$ however, the beneficial effects of targeting intensive glycaemic control were not as clear in our review as in the DCCT/ EDIC. ${ }^{3-46}$ The definition of retinopathy varied among trials in our meta-analysis (web appendix 4), and duration of follow-up also (table 1). We found no consistent statistically significant effect on retinopathy (ie, the significance was present in the fixed effect, but not the random effect model) when meta-analysing all available randomised clinical trials of targeting intensive versus conventional glucose control in patients with type 1 diabetes mellitus. This is noteworthy, because retinopathy (sustained 3-step progression) was the primary outcome of DCCT. However, there was a baseline imbalance in favour of the intensive group for retinopathy in the secondary prevention cohort in DCCT — which was adjusted for in the analyses from the trial. Nonetheless, baseline imbalances can result from insufficient randomisation and can be problematic for the interpretation, in particular for the primary outcome-even if adjusted for.

Since the result of the DCCT/EDIC, ${ }^{3-46}$ only a few small trials have been investigating the effect of intensive glycaemic control. Our results suggest that more trials are needed before the results from the DCCT/EDIC can be confirmed. The difficulties in applying the results from DCCT $/ \mathrm{EDIC}^{3-46}$ to a clinical context are many. The participants had a short duration of type 1 diabetes mellitus (mean 6 years), were without cardiovascular diseases, and were young (mean age 27 years). Besides, several of the trials, including DCCT/EDIC, ${ }^{3-46}$ had a very loose approach to the conventional treatment, that is, only to avoid symptoms of hyperglycaemia. Only one of the trials $(n=99)$ randomised participants to a $\mathrm{HbA}_{1 \mathrm{c}}$ target of $7 \%$ in the intensive group-the target recommended by one of the major guidelines by The American Diabetes Association. ${ }^{47}$ Trial investigators might conclude that trials randomising participants to a looser glycaemic control are unethical given the positive effect on the microvascular complications in the DCCT/ EDIC. ${ }^{3-46}$ The positive effect on the microvascular outcomes in DCCT/EDIC was to a large extent delivered by surrogate markers, including the primary outcome, 3-step progression in retinopathy, and it should be kept in mind that, like in our meta-analysis, no statistically significant effect was shown for the hard clinical microvascular outcome, end-stage renal disease. Furthermore, in our meta-analysis, data on the most important outcomes such as all-cause mortality and cardiovascular mortality showed no effect of targeting an intensive glycaemic control. It can therefore be questioned whether a new trial would be unethical. Owing to the lack of data in the relevant trials, it is hard to formulate guidelines. We advocate physicians to consider our results when weighing the benefits and harms of raising/lowering the glycaemic target in patients with type 1 diabetes mellitus.

\section{CONCLUSIONS}

We found no statistically significant effect of targeting an intensive glycaemic control compared with conventional glycaemic control on all-cause and cardiovascular mortality. However, we did find indications of a possible positive effect of targeting intensive glycaemic control on macrovascular complications as a composite outcome and on nephropathy. On the other hand, we found statistically significant more severe hypoglycaemia and increase in body mass index in the intensively treated patients. Notably, we found no consistent effect on retinopathy and ketoacidosis. The trial sequence analysis showed that the decision to choose a glycaemic intervention target is based on sparse data. In addition, most trials had poor bias control, and there was a severe lack of reporting on patient relevant outcomes. More randomised clinical trials with low bias risk are needed to conclude whether targeting an intensive glycaemic control is superior or not for improving patient relevant outcomes in patients with type 1 diabetes mellitus.

\section{Author affiliations}

${ }^{1}$ Copenhagen Trial Unit, Centre for Clinical Intervention Research, Department 7812, Rigshospitalet, Copenhagen University Hospital, Copenhagen, Denmark ${ }^{2}$ Department of Medicine F, Gentofte Hospital, Gentofte, Denmark ${ }^{3}$ Cochrane Hepato-Biliary Group, Copenhagen Trial Unit, Centre for Clinical Intervention Research, Department 7812, Rigshospitalet, Copenhagen University Hospital, Copenhagen, Denmark

${ }^{4}$ Department of Endocrinology, Rigshospitalet, Copenhagen University Hospital, Copenhagen, Denmark

Acknowledgements The authors thank Sarah Klingenberg, the trials search co-ordinator of the Cochrane Hepato-Biliary Group, for her assistance in developing the search strategy. They also thank Victoria Franklin for answering their requests for information on trials and Søren S Lund for his valuable suggestions and contrubutions during the development of this review. Søren S Lund is an employee of Boehringer Ingelheim, Germany. Søren S Lund's contribution was his alone and does not necessarily reflect the official position of Boehringer Ingelheim.

Contributors PK developed the protocol, was responsible for the searches, selected trials, extracted data, assessed risk of bias of trials, did the data analysis, and developed the final review. BH developed the protocol, was responsible for the searches, selected trials, extracted data, assessed risk of bias of trials, and developed the final review. BG developed the protocol, selected trials, extracted data, assessed risk of bias of trials, did the data analysis, and developed the final review. CG developed the protocol, selected trials, advised on statistical methods, and developed the final review. TA developed the initial idea for the review, developed the protocol, and developed the final review. AV developed the protocol, and developed the final review. JW developed the protocol, advised on statistical methods, and developed the final review. All authors read and approved the final manuscript.

Funding The study was funded by The Copenhagen Trial Unit, Centre for Clinical Intervention Research, Rigshospitalet, Denmark. 
Competing interests PK, AV, and TA have reported equity in Novo Nordisk A/S. AV have received fees from Novo Nordisk A/S for speaking.

Provenance and peer review Not commissioned; externally peer reviewed.

Data sharing statement No additional data are available.

Open Access This is an Open Access article distributed in accordance with the Creative Commons Attribution Non Commercial (CC BY-NC 3.0) license, which permits others to distribute, remix, adapt, build upon this work noncommercially, and license their derivative works on different terms, provided the original work is properly cited and the use is non-commercial. See: http:// creativecommons.org/licenses/by-nc/3.0/

\section{REFERENCES}

1. Klein BE, Klein R, McBride PE, et al. Cardiovascular disease, mortality, and retinal microvascular characteristics in type 1 diabetes: Wisconsin epidemiologic study of diabetic retinopathy. JAMA Intern Med 2004:164:1917-24.

2. Lind M, Bounias I, Olsson M, et al. Glycaemic control and incidence of heart failure in 20985 patients with type 1 diabetes: an observational study. Lancet 2011;378:140-6.

3. Danis RP, Davis MD, White N, et al. 10-Year retinopathyoutcomes in the EDIC cohort: effect of the original DCCT treatment group assignment. IOVS 2005

4. The DCCT Research Group. Adverse events and their association with treatment regimens in the diabetes control and complications trial. Diabetes Care 1995;18:1415-27.

5. Dailey GE, Boden $\mathrm{GH}$, Creech $\mathrm{RH}$, et al. Effects of pulsatile intravenous insulin therapy on the progression of diabetic nephropathy. Metabolism 2000;49:1491-5.

6. De Boer IH, Sachs MC, Cleary PA, et al. Circulating vitamin D metabolites and kidney disease in type 1 diabetes. J Clin Endocrinol Metab 2012;97:4780-8.

7. The DCCT Research Group. Progression of retinopathy with intensive versus conventional treatment in the Diabetes Control and Complications Trial. Ophthalmology 1995;102:647-61.

8. The DCCT Research Group. Diabetes Control and Complications Trial (DCCT). Update. Diabetes Care 1990;13:427-33

9. The DCCT Research Group. The effect of intensive diabetes treatment on the progression of diabetic retinopathy in insulin-dependent diabetes mellitus. The Diabetes Control and Complications Trial. Arch Ophthalmol 1995;113:36-51.

10. The DCCT Research Group. Early worsening of diabetic retinopathy in the diabetes control and complications trial. Arch Ophthalmol 1998;116:874-86.

11. DCCT Research Group. Protocol (Full-Scale Clinical Trial-Phase III) for the Diabetes Control and Complications Trial, 1987. http://www2. bsc.gwu edu/bsc/oneproj.php?pkey=5 (accessed 5 Apr 2013).

12. The DCCT Research Group. Effect of intensive therapy on the development and progression of diabetic nephropathy in the Diabetes Control and Complications Trial. Kidney Int 1995;47:1703-20.

13. The DCCT Research Group. Hypoglycemia in the diabetes control and complications trial. Diabetes 1997;46:271-86.

14. The DCCT Research Group. Effect of intensive diabetes management on macrovascular events and risk factors in the Diabetes Control and Complications Trial. Am J Cardiol 1995;75:894-903.

15. The DCCT Research Group. Influence of intensive diabetes treatment on body weight and composition of adults with type 1 diabetes in the Diabetes Control and Complications Trial. Diabetes Care 2001:24:1711-21.

16. The DCCT Research Group. Influence of intensive diabetes treatment on quality-of-life outcomes in the diabetes control and complications trial. Diabetes Care 1996;19:195-203.

17. The DCCT Research Group. The relationship of glycemic exposure $(\mathrm{HbA1c})$ to the risk of development and progression of retinopathy in the diabetes control and complications trial. Diabetes 1995;44:968-83.

18. [No authors listed]. The relationship of glycemic exposure (HbA1c) to the risk of development and progression of retinopathy in the diabetes control and complications trial. Diabetes 1995;44:968-83.

19. The DCCT Research Group. Weight gain associated with intensive therapy in the diabetes control and complications trial. Diabetes Care 1988;11:567-73.

20. Hubbard LD, Sun W, Cleary PA, et al. Comparison of digital and film grading of diabetic retinopathy severity in the diabetes control and complications trial/epidemiology of diabetes interventions and complications study. Arch Ophthalmol 2011;129:718-26.

21. Younes N, Cleary PA, Steffes MW, et al. Comparison of urinary albumin-creatinine ratio and albumin excretion rate in the Diabetes Control and Complications Trial/Epidemiology of Diabetes Interventions and Complications study. Clin J Am Soc Nephrol 2010:5:1235-42.

22. Keen H. The Diabetes Control and Complications Trial (DCCT). Health Trends 1994;26:41-3.

23. Kilpatrick ES, Rigby AS, Atkin SL. Variability in the relationship between mean plasma glucose and HbA1c: implications for the assessment of glycemic control. Clin Chem 2007;53:897-901.

24. Genuth S. Insights from the diabetes control and complications trial/epidemiology of diabetes interventions and complications study on the use of intensive glycemic treatment to reduce the risk of complications of type 1 diabetes. Endocr Pract 2006;12:34-44.

25. Crofford OB, Genuth S, Baker L. Diabetes Control and Complications Trial (DCCT): results of feasibility study. Diabetes Care 1987;10:1-19.

26. Lachin JM, Genuth S, Nathan DM, et al. Effect of glycemic exposure on the risk of microvascular complications in the diabetes control and complications trial-revisited. Diabetes 2008:57:995-1001.

27. The DCCT Research Group. Effect of intensive diabetes treatment on the development and progression of long-term complications in adolescents with insulin-dependent diabetes mellitus: Diabetes Control and Complications Trial. J Pediatr 1994;125:177-88.

28. Writing Team for The DCCT/EDIC Research Group. Effect of intensive therapy on the microvascular complications of type 1 diabetes mellitus. JAMA 2002;287:2563-9.

29. White $\mathrm{NH}$, Sun $\mathrm{W}$, Cleary PA, et al. Effect of prior intensive therapy in type 1 diabetes on 10-year progression of retinopathy in the DCCT/EDIC: comparison of adults and adolescents. Diabetes 2010;59:1244-53.

30. Liu Z, Poole C, Herring AH, et al. Estimation of acute effects of severe hypoglycemia on subsequent episodes in type 1 diabetes. http:// www.pharmacoepi.org/meetings/27thconf/ (accessed 12 Feb 2013).

31. Lin J, Glynn RJ, Rifai N, et al. Inflammation and progressive nephropathy in type 1 diabetes in the diabetes control and complications trial. Diabetes Care 2008;31:2338-43.

32. The DCCT Research Group. The Diabetes Control and Complications Trial (DCCT). Design and methodologic considerations for the feasibility phase. Diabetes 1986;35:530-45.

33. Boer $\mathrm{IH}$, Sun W, Cleary PA, et al. Intensive diabetes therapy and glomerular filtration rate in type 1 diabetes. $N$ Engl J Med 2011;365:2366-76.

34. Nathan DM, Cleary PA, Backlund JY, et al. Intensive diabetes treatment and cardiovascular disease in patients with type 1 diabetes. N Engl J Med 2005;353:2643-53.

35. Allison SJ. Intensive glucose control in type 1 diabetes mellitus reduces risk of GFR loss. Nat Rev Endocrinol 2012;8:65.

36. Boer IH, Rue TC, Cleary PA, et al. Long-term renal outcomes of patients with type 1 diabetes mellitus and microalbuminuria: an analysis of the Diabetes Control and Complications Trial/ Epidemiology of Diabetes Interventions and Complications cohort. Arch Intern Med 2011;171:412-20.

37. The DCCT/EDIC Research Group. Modern-day clinical course of type 1 diabetes mellitus after 30 years' duration: the diabetes control and complications trial/epidemiology of diabetes interventions and complications and Pittsburgh epidemiology of diabetes complications experience (1983-2005). Arch Intern Med 2009;169:1307-16.

38. White $\mathrm{NH}$, Sun W, Cleary PA, et al. Prolonged effect of intensive therapy on the risk of retinopathy complications in patients with type 1 diabetes mellitus: 10 years after the Diabetes Control and Complications Trial. Arch Ophthalmol 2008;126:1707-15.

39. Kilpatrick ES, Rigby AS, Goode K, et al. Relating mean blood glucose and glucose variability to the risk of multiple episodes of hypoglycaemia in type 1 diabetes. Diabetologia 2007;50:2553-61.

40. The DCCT/EDIC Research Group. Retinopathy and nephropathy in patients with type 1 diabetes four years after a trial of intensive therapy. N Engl J Med 2000;342:381-9.

41. Nathan DM. Sustained effect of intensive treatment of type 1 diabetes mellitus on development and progression of diabetic nephropathy: the Epidemiology of Diabetes Interventions and Complications (EDIC) Study. JAMA 2003;290:2159-67.

42. Cleary PA, Orchard TJ, Genuth S, et al. The effect of intensive glycemic treatment on coronary artery calcification in type 1 diabetic participants of the Diabetes Control and Complications Trial/Epidemiology of Diabetes Interventions and Complications (DCCT/EDIC) Study. Diabetes 2006;55:3556-65. 
43. Anon. Type 1 diabetes: benefits of intensive insulin therapy. Patients should control blood glucose strictly. Prescrire Int 2002;11:61.

44. EDIC Data Coordinating Center. EDIC Protocol, Epidemiology of Diabetes Interventions and Complications Continuing Follow-Up. 2012. http://www2.bsc.gwu.edu/bsc/docs/edicprot\%282\%29.pdf (accessed 12 Feb 2013).

45. Purnell JQ, John E, Hokanson Cleary PA, et al. The effect of excess weight gain with intensive diabetes mellitus treatment on cardiovascular disease risk factors and atherosclerosis in type 1 diabetes mellitus: results from the Diabetes Control and Complications Trial/Epidemiology of Diabetes Interventions and Complications Study (DCCT/EDIC) study. Circulation 2013;127:180-7.

46. Rogers DG. The effect of intensive treatment of diabetes on the development and progression of long-term complications in insulin-dependent diabetes mellitus. Clin Pediatr 1994;33:378.

47. American Diabetes Association. Tight Diabetes Control. http://www. diabetes.org/living-with-diabetes/treatment-and-care/blood-glucosecontrol/tight-diabetes-control.html (accessed 24 Jan 2013).

48. Ceriello A, Colagiuri S. International diabetes federation guideline for management of postmeal glucose: a review of recommendations. Diabet Med 2008:25:1151-6.

49. Egger M, Davey Smith G, Stettler C, et al. Risk of adverse effects of intensified treatment in insulin-dependent diabetes mellitus: a meta-analysis. Diabet Medicine 1997;14:919-28.

50. Microalbuminuria Collaboration Group. Microalbuminuria in type I diabetic patients. Diabetes Care 1992;15:495-501.

51. Microalbuminuria Collaborative Study Group. Intensive therapy and progression to clinical albuminuria in patients with insulin dependent diabetes mellitus and microalbuminuria. BMJ 1995:311:973-7.

52. Higgins J, Green S. Cochrane Handbook for Systematic Reviews of Interventions Version 5.1.0 [updated March 2011]. The Cochrane Collaboration 2011. http://www.cochrane-handbook.org

53. Kähler P, Grevstad B, Almdal T, et al. Targeting intensive glycaemic control versus targeting conventional glycaemic control for type 1 diabetes mellitus: systematic review with meta-analyses and trial sequential analyses of randomised clinical trials. Prospero 2013:CRD42013003801. http://www.crd.york.ac.uk/Prospero/

54. Review Manager (RevMan) [Computer program]. Version 5.2. Copenhagen: The Nordic Cochrane Centre. The Cochrane Collaboration, 2012

55. Demets $\mathrm{D}$. Methods for combining randomized clinical trials: strengths and limitations. Stat Med 1987:6:341-50.

56. DerSimonian R, Laird N. Meta-analysis in clinical trials. Control Clin Trials 1986;7:177-88.

57. Stettler C, Allemann S, Juni P, et al. Glycemic control and macrovascular disease in types 1 and 2 diabetes mellitus: meta-analysis of randomized trials. Am Heart J 2006;152:27-38.

58. Egger M, Davey Smith G, Stettler C, et al. Risk of adverse effects of intensified treatment in insulin-dependent diabetes mellitus: a meta-analysis. Diabet Med 1997;14:919-28.

59. Lawson ML, Gerstein HC, Tsui E, et al. Effect of intensive therapy on early macrovascular disease in young individuals with type 1 diabetes: A systematic review and meta-analysis. Diabetes Care 1999;22:35-9.

60. Mattila TK, de Boer A. Influence of intensive versus conventional glucose control on microvascular and macrovascular complications in type 1 and 2 diabetes mellitus. Drugs 2010;70:2229-45.

61. Wang PH, Lau J, Chalmers TC. Meta-analysis of effects of intensive blood glucose control on late complications of type I diabetes mellitus. Lancet 1993;341:1306-9.

62. Thorlund K, Imberger G, Walsh M, et al. The number of patients and events required to limit the risk of overestimation of intervention effects in meta-analysis-a simulation study. PLOS ONE 2011;6: e25491.

63. Wetterslev J, Thorlund K, Brok J, et al. Trial sequential analysis may establish when firm evidence is reached in cumulative meta-analysis. J Clin Epidemiol 2008;61:64-75.

64. Wetterslev J, Thorlund K, Brok J, et al. Estimating required information size by quantifying diversity in random-effects model meta-analyses. BMC Med Res Methodol 2009;9:86.

65. Brok J, Thorlund K, Gluud C, et al. Trial sequential analysis reveals insufficient information size and potentially false positive results in many meta-analyses. J Clin Epidemiol 2008;61:763-9.

66. Higgins J, Whitehead A, Simmonds M. Sequential methods for random-effects meta-analysis. Stat Med 2011;30:903-21.

67. The Copenhagen Trial Unit. Trial sequential analysis [computer program]. Version 0.9 beta. The Copenhagen Trial Unit, Centre for Clinical Intervention Research, 2011. http://www.ctu.dk/tsa
68. Madsbad S, Krarup T, Faber O, et al. The transient effect of strict glycaemic control on B-cell function in newly diagnosed type 1 (insulin-dependent) diabetic patients. Diabetologia 1982;22:16-20.

69. Linn T, Mann M, Bretzel RG, et al. Randomised prospective study for the effect of therapy on residual beta cell function in type-1 diabetes mellitus. BMC Endocr Disord 2003;3:5.

70. Feldt-Rasmussen B, Mathiesen ER, Hegedüs L, et al. Kidney function during 12 months of strict metabolic control in insulin-dependent diabetic patients with incipient nephropathy. N Engl J Med 1986;314:665-70.

71. Feldt-Rasmussen B, Mathiesen ER, Deckert T. Effect of two years of strict metabolic control on progression of incipient nephropathy in insulin-dependent diabetes. Lancet 1986;2:1300-4.

72. Feldt-Rasmussen B, Mathiesen ER, Jensen T, et al. Effect of improved metabolic control on loss of kidney function in type 1 (insulin-dependent) diabetes patients: An update of the Steno studies. Diabetologia 1991;34:164-70.

73. Brinchmann-Hansen O, Dahl-Jørgensen K, Hanssen KF, et al. Effects of intensified insulin treatment on various lesions of diabetic retinopathy. Am J Ophthalmol 1985;100:644-53.

74. Brinchmann-Hansen O, Dahl-Jørgensen K, Hanssen KF, et al. Effects of intensified insulin treatment on retinal vessels in diabetic patients. Br J Ophthalmol 1988;72:666-73.

75. Brinchmann-Hansen O, Dahl-Jørgensen K, Hanssen KF, et al. Oscillatory potentials, macular recovery time, and diabetic retinopathy through 3 years of intensified insulin treatment Ophthalmology 1988:1358-66.

76. Brinchmann-Hansen O, Dahl-Jørgensen K, Hanssen KF, et al. The response of diabetic retinopathy to 41 months of multiple insulin injections, insulin pumps, and conventional insulin therapy. Arch Ophthalmol 1988;106:1242-6.

77. Dahl-Jørgensen K, Brinchmann-Hansen O, Hanssen KF, et al. Effect of near normoglycaemia for two years on progression of early diabetic retinopathy, nephropathy, and neuropathy: the Oslo study. BMJ 1986;293:1195-9.

78. Dahl-Jørgensen K, Brinchmann-Hansen O, Hanssen KF, et al. Rapid tightening of blood glucose control leads to transient deterioration of retinopathy in insulin dependent diabetes mellitus: the Oslo study. BMJ 1985;290:811-15.

79. Hanssen KF, Brinchmann-Hansen O, Dahl-Jørgensen K, et al. Effect of intensive treatment on diabetic retinopathy. Journ Annu Diabetol Hotel Dieu 1988:167-73.

80. Amthor KF, Dahl-Jørgensen K, Berg TJ, et al. The effect of 8 years of strict glycaemic control on peripheral nerve function in IDDM patients: the Oslo Study. Diabetologia 1994;37:579-84.

81. Service FJ, Daube JR, O'Brien PC, et al. Effect of blood glucose control on peripheral nerve function in diabetic patients. Mayo Clin Proc 1983:58:283-9.

82. Verrillo A, Teresa A, Martino C, et al. Long-term correction of hyperglycemia and progression of retinopathy in insulin dependent diabetes. A five-year randomized prospective study. Diabetes Res 1988;8:71-6

83. Barbosa J, Steffes MW, Sutherland DE, et al. Effect of glycemic control on early diabetic renal lesions. A 5-year randomized controlled clinical trial of insulin-dependent diabetic kidney transplant recipients. JAMA 1994;272:600-6.

84. Barbosa J, Connett J, Fryd D, et al. The Minnesota diabetes complications clinical trial. The first three years. Acta Diabetol Lat 1983;20:165-71.

85. Hung J, Menth L, Thompson MJ, et al. The Minnesota Diabetes Complications Clinical Trial cognitive functions under long-term maximized and standard metabolic controls. Diabete Metab 1984; 10:48-51.

86. Barbosa J, Johnson S. Severe hypoglycemia during maximized insulin treatment of diabetes in a randomized clinical trial. Diabetes Care 1983;6:62-3.

87. Reichard P. Are there any glycemic thresholds for the serious microvascular diabetic complications? J Diabetes Complications 1995;9:25-30.

88. Reichard P, Sule J, Rosenqvist U. Capillary loss and leakage after five years of intensified insulin treatment in patients with insulin-dependent diabetes mellitus. Ophthalmology 1991;98:1587-93.

89. Reichard P, Pihl M, Rosenqvist U, et al. Complications in IDDM are caused by elevated blood glucose level: the Stockholm diabetes intervention study (SDIS) at 10-year follow up. Diabetologia 1996;39:1483-8.

90. Jensen-Urstad KJ, Reichard PG, Rosfors JS, et al. Early atherosclerosis is retarded by improved long-term blood glucose control in patients with IDDM. Diabetes 1996;45:1253-8. 
91. Reichard P, Berglund A, Britz A, et al. Hypoglycaemic episodes during intensified insulin treatment: increased frequency but no effect on cognitive function. $J$ Intern Med 1991;229:9-16.

92. Johansson J, Reichard P, Jensen-Urstad K, et al. Influence of glucose control, lipoproteins, and haemostasis function on brachial endothelial reactivity and carotid intima-media area, stiffness and diameter in type 1 diabetes mellitus patients. Eur J Clin Invest 2003;33:472-9.

93. Reichard $\mathrm{P}$, Berglund $\mathrm{B}$, Britz $\mathrm{A}$, et al. Intensified conventional insulin treatment retards the microvascular complications of insulin-dependent diabetes mellitus (IDDM): the Stockholm Diabetes Intervention Study (SDIS) after 5 years. J Intern Med 1991;230:101-8.

94. Reichard P, Britz A, Carlsson P, et al. Metabolic control and complications over 3 years in patients with insulin dependent diabetes (IDDM): the Stockholm Diabetes Intervention Study (SDIS). J Intern Med 1990;228:511-17.

95. Reichard $\mathrm{P}$, Rosenqvist $\mathrm{U}$. Nephropathy is delayed by intensified insulin treatment in patients with insulin-dependent diabetes mellitus and retinopathy. J Intern Med 1989;226:81-7.

96. Reichard P, Pihl M. Mortality and treatment side-effects during long-term intensified conventional insulin treatment in the Stockholm Diabetes Intervention Study. Diabetes 1994;43:313-17.

97. Reichard P, Britz A, Cars I, et al. The Stockholm Diabetes Intervention Study (SDIS): 18 months' results. Acta Med Scand 1988;224:115-22.

98. Reichard P, Nilsson BY, Rosenqvist U. The effect of long-term intensified insulin treatment on the development of microvascular complications of diabetes mellitus. N Engl J Med 1993;329:304-9.

99. Savović J, Jones H, Altman D, et al. Influence of reported study design characteristics on intervention effect estimates from randomized, controlled trials. Ann Intern Med 2012;157:429-38.

100. Wood L, Egger M, Gluud L, et al. Empirical evidence of bias in treatment effect estimates in controlled trials with different interventions and outcomes: meta-epidemiological study. BMJ 2008;336:601-5.

101. Shah SC, Malone JI, Simpson NE. A randomized trial of intensive insulin therapy in newly diagnosed insulin-dependent diabetes mellitus. N Engl J Med 1989;320:550-4.

102. Hershey $\mathrm{T}$, Bhargava N, Sadler M, et al. Conventional versus intensive diabetes therapy in children with type 1 diabetes: effects on memory and motor speed. Diabetes Care 1999;22:1318-24.

103. White $\mathrm{NH}$. Intensive diabetes therapy is effective in children. $A m$ Fam Physician 1994;50:407.

104. Franklin VL, Khan F, Kennedy G, et al. Intensive insulin therapy improves endothelial function and microvascular reactivity in young people with type 1 diabetes. Diabetologia 2008;46:353-60.

105. Perlman K, Ehrlich RM, Filler RM, et al. Sustained normoglycemia in newly diagnosed type I diabetic subjects. Short-term effects and one-year follow-up. Diabetes 1984;33:995-1001.

106. Fullerton B, Jeitler K, Seitz M, et al. Intensive glucose control versus conventional glucose control for type 1 diabetes mellitus. Cochrane Database Syst Rev 2014;2:CD009122.

107. Bangstad HJ, Kofoed-Enevoldsen A, Dahl-Jørgensen K, et al. Glomerular charge selectivity and the influence of improved blood glucose control in type 1 (insulin-dependent) diabetic patients with microalbuminuria. Diabetologia 1992;35:1165-9.

108. Lehto S, Rönnemaa T, Pyörälä K, et al. Poor glycemic contro predicts coronary heart disease events in patients with type 1 diabetes without nephropathy. Arterioscler Thromb Vasc Biol 1999;19:1014-19.

109. Jakobsen J, Gluud C. The necessity of randomized clinical trials. Br J Med Med Res 2013;3:1453-68.

110. Linn T, Ortac K, Laube $\mathrm{H}$, et al. Intensive therapy in adult insulin-dependent diabetes mellitus is associated with improved insulin sensitivity and reserve: a randomized, controlled, prospective study over 5 years in newly diagnosed patients. Metabolism 1996;45:1508-13.

111. Holman RR, Dornan TL, Mayon-White V, et al. Prevention of deterioration of renal and sensory-nerve function by more intensive management of insulin-dependent diabetic patients. A two-year randomised prospective study. Lancet 1983;1:204-8.

112. Champion M, Bending J, Rodger N, et al. Recruitment randomization, and baseline characteristics of the treatment groups. Diabetes 1985;34:13-16.

113. Champion M, Keen $\mathrm{H}$, Pickup J, et al. Origin and design of the Kroc Collaborative Study. Diabetes 1985;34:5-12.

114. Lawson P, Home PD, Bergenstal R. Observations on blood lipid and intermediary metabolite concentrations during conventional insulin treatment or CSII. Diabetes 1985;34:27-30.

115. Bending J, Viberti G, Bilous R, et al. Eight-month correction of hyperglycemia in insulin-dependent diabetes mellitus is associated with a significant and sustained reduction of urinary albumin excretion rates in patients with microalbuminuria. Diabetes 1985;34:69-73.

116. The Kroc Collaboration Study Group. Diabetic retinopathy after two years of intensified insulin treatment. Follow-up of the Kroc Collaborative Study. JAMA 1988;260:37-41.

117. The Kroc Collaborative Study Group. Blood glucose control and the evolution of diabetic retinopathy and albuminuria. A preliminary multicenter trial. N Engl J Med 1984;311:365-72.

118. Callaghan BC, Little AA, Feldman EL, et al. Enhanced glucose control for preventing and treating diabetic neuropathy. Cochrane Database Syst Rev 2012;6:CD007543.

119. Lauritzen T, Frost-Larsen K, Larsen HW, et al. Two-year experience with continuous subcutaneous insulin infusion in relation to retinopathy and neuropathy. Diabetes 1985;34: 74-9.

120. Lauritzen T, Frost LK, Larsen HW, et al. Metabolic regulation, retinal function and retinal morphology during one-year treatment with continuous subcutaneous insulin infusion (CSII) and conventional therapy (CT). A randomized prospective study. Acta Endocrinol Suppl 1982;100:41.

121. Steno study group. Effect of 6 months of strict metabolic control on eye and kidney function in insulin-dependent diabetics with background retinopathy. Lancet 1982;1:121-4. 


\section{Correction}

Kähler P, Grevstad B, Almdal T, et al. Targeting intensive versus conventional glycaemic control for type 1 diabetes mellitus: a systematic review with meta-analyses and trial sequential analyses of randomised clinical trials. BMJ Open 2014;4:e004806. The list of authors should read:

Pernille Kähler, ${ }^{1}$ Berit Grevstad, ${ }^{1}$ Thomas Almdal, ${ }^{2}$ Christian Gluud, ${ }^{1}{ }^{3}$ Jørn Wetterslev, ${ }^{1}$ Søren Søgaard Lund, ${ }^{4}$ Allan Vaag, ${ }^{5}$ Bianca Hemmingsen ${ }^{1}$

${ }^{1}$ Copenhagen Trial Unit, Centre for Clinical Intervention Research, Department 7812, Rigshospitalet, Copenhagen University Hospital, Copenhagen, Denmark

${ }^{2}$ Department of Medicine F, Gentofte Hospital, Gentofte, Denmark

${ }^{3}$ Cochrane Hepato-Biliary Group, Copenhagen Trial Unit, Centre for Clinical Intervention Research, Department 7812, Rigshospitalet, Copenhagen University Hospital, Copenhagen, Denmark

${ }^{4}$ Boehringer Ingelheim Pharma GmbH \& Co. KG, Ingelheim, Germany

${ }^{5}$ Department of Endocrinology, Rigshospitalet, Copenhagen University Hospital, Copenhagen, Denmark

The Acknowledgements and Contributors sections should read:

Acknowledgements The authors thank Sarah Klingenberg, the trials search co-ordinator of the Cochrane Hepato-Biliary Group, for her assistance in developing the search strategy. They also thank Victoria Franklin for answering our requests for information on trials.

Contributors PK developed the protocol, was responsible for the searches, selected trials, extracted data, assessed risk of bias of trials, did the data analysis, and developed the final review. $\mathrm{BH}$ developed the protocol, was responsible for the searches, selected trials, extracted data, assessed risk of bias of trials, and developed the final review. BG developed the protocol, selected trials, extracted data, assessed risk of bias of trials, did the data analysis, and developed the final review. CG developed the protocol, selected trials, advised on statistical methods, and developed the final review. TA developed the initial idea for the review, developed the protocol, and developed the final review. AV developed the protocol, and developed the final review. JW developed the protocol, advised on statistical methods, and developed the final review. SSL contributed substantially to the interpretation of data and revised the manuscript critically for important intellectual content. All authors read and approved the final manuscript.

Competing interests PK, AV, SSL and TA have reported equity in Novo Nordisk A/S. SSL owns shares in dynamically traded investment funds, which may own stocks from pharmaceutical companies. AV have received fees from Novo Nordisk A/S for speaking. SSL is an employee of Boehringer Ingelheim Pharma GmbH \& Co. KG, Ingelheim, Germany; his contribution was his alone and does not necessarily reflect the official position of Boehringer Ingelheim.

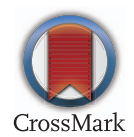

BMJ Open 2015;5:004806corr1. doi:10.1136/bmjopen-2014-004806corr1 